\title{
Landau fluid closures with nonlinear large-scale finite Larmor radius corrections for collisionless plasmas
}

\author{
P. L. Sulem $†$ and T. Passot \\ Laboratoire J.L. Lagrange, Université de Nice-Sophia Antipolis, CNRS, Observatoire de la Côte d'Azur, \\ Boulevard de l'Observatoire, CS 34229, 06304 Nice Cedex 4, France \\ (Received 22 May 2014; revised 28 July 2014; accepted 4 August 2014; \\ first published online 5 September 2014)
}

With the aim to develop a tool for simulating turbulence in collisionless magnetized plasmas, fluid models retaining low-frequency kinetic effects such as Landau damping and finite Larmor radius (FLR) corrections are discussed. It turns out that, in the absence of ion-cyclotron resonance, the dispersion and damping of kinetic Alfvén waves at scales as small as a fraction of the ion Larmor radius are accurately reproduced when using fluid estimates of the non-gyrotropic moments, at leading-order within a large-scale asymptotics. Differently, evaluations based on the low-frequency linear kinetic theory are necessary in regimes of large temperature anisotropies, and in particular in the presence of the mirror instability. Combining both descriptions leads to a new Landau fluid model retaining large-scale FLR nonlinearities, while reproducing the linear dynamics of low-frequency modes at the sub-ionic scales.

\section{Introduction}

In many instances, magnetohydrodynamics (MHD) or its dispersive extension including the Hall effect (Hall-MHD) cannot provide a satisfactory description of collisionless or weakly collisional plasmas, in part due to the lack of realistic wave damping. On the other hand, especially in the turbulent regime, a fully kinetic description requires huge computational resources and is still difficult to implement, even on the present-day most powerful computers. This situation motivates making efforts to extend the fluid description to magnetized plasmas by retaining kinetic effects such as Landau damping and finite Larmor radius (FLR) corrections at scales comparable to or smaller than the ion gyroradius. As with the gyrokinetic theory (see e.g. Howes et al. (2006)), the aim is to capture the low-frequency dynamics, when ion-cyclotron resonances can be overlooked.

Linear Landau damping was first retained within a fluid description of a magnetized plasma by Snyder et al. (1997) who developed a so-called Landau fluid model for the MHD scales by performing a closure of the hierarchy derived from the drift kinetic equation. The fourth-rank cumulants of the various particle species, or in a simplified formulation, the heat fluxes, are expressed in terms of lower rank moments in a way consistent with the low-frequency linear kinetic theory, up to the replacement of the plasma dispersion function by suitable Padé approximants. This description

$\dagger$ Email address for correspondence: sulem@oca.eu 
involves dynamical equations for the parallel and perpendicular pressures of the ions and the electrons, and thus permits the development of temperature anisotropies. Another step in the development of Landau fluid models was made by supplementing FLR corrections. Their effect is to be retained when considering scales close to the ion gyroradius. An example is provided by the computation of non-homogeneous equilibrium configurations serving as initial conditions for kinetic simulations of magnetic reconnection with shear flows (Cerri et al. 2013). FLR-corrections can be calculated in two ways. A first approach consists in solving the equations for the non-gyrotropic parts of the pressure or heat flux tensors, perturbatively with respect to a small parameter measuring the space and time scale separation between the ion gyromotion and the considered phenomena (Macmahon 1965; Ramos 2005). When limited to the linear regime (Goswami et al. 2005; Passot et al. 2012), this approach is easily implemented, even when retaining second order contributions, but the general formulation, that has the main advantage of being asymptotically exact, becomes excessively intricate when going beyond the leading order. Another approach consists in expressing all the non-gyrotropic moments directly from the low-frequency kinetic theory, again modeling the plasma dispersion function with appropriate Padé approximants (Passot and Sulem 2007; Passot et al. 2012). This description retains all the scales in the directions quasi-transverse to the ambient magnetic field, thus reproducing the linear properties of kinetic Alfvén waves, such as their dispersion relation, polarization and magnetic compressibility (Hunana et al. 2013a,b). It also provides a precise description of the mirror instability and of its arrest at small scales. This so-called FLR-Landau fluid model was recently implemented for three-dimensional simulations of both Alfvén and mirror turbulences (Passot et al. 2014). It is noticeable that in these simulations, as long as the turbulence level is sufficiently low, ion and electron Landau damping provide a sufficient dissipation for numerical simulations to be stable, with no need for filtering nor artificial dissipation. Power law spectra, similar to those observed in satellite data (Alexandrova et al. 2008; Sahraoui et al. 2009, 2010), develop in the so-called dissipation range, down to the smallest resolved scale. Trying to benefit from the advantages of both FLR descriptions suggests a matching procedure, resulting in an asymptotically exact nonlinear description at large scales and a linearly correct modeling of the lowfrequency small-scale fluctuations.

The present paper is organized as follows. Section 2 briefly reviews the fluid hierarchy derived from the Vlasov-Maxwell equations, mainly to specify the notations. Section 3 discusses a fluid model involving the leading-order FLR contributions to the gyrotropic pressure and heat flux tensors, within a large-scale asymptotics (see e.g. Ramos (2005)), to which we supplement fluid-hierarchy closures retaining Landau damping, either at the level of the third or of the fourth-rank moments. Although these closures are based on the linear kinetic theory, they nevertheless must take into account the curvature of the magnetic field lines, in order to prevent spurious instabilities in the nonlinear regime (Passot et al. 2014). In Sec. 4, the resulting models are assessed at the level of the dispersion and damping of linear kinetic Alfvén waves, which turn out to be accurately captured, even at scales significantly smaller than guaranteed by the asymptotic ordering, at least when the temperature anisotropy is not too strong. When the temperature anisotropy is large enough for micro-instabilities such as the mirror instability to develop, a more accurate description of the sub-ion scales, as provided by the FLR-Landau fluid model discussed in Passot and Sulem (2007) and Passot et al. (2012), is required. After revisiting this model in Sec. 5.1, we discuss in Sec. 5.2 a new fluid model, resulting from a matching of the large-scale 
nonlinear asymptotics of Sec. 3 with the small-scale kinetic-based approach of Sec. 5.1. Section 6 summarizes the main conclusions.

\section{The fluid hierarchy}

We consider a proton-electron plasma and denote the particle species by the subscript $r$, which may refer to the ions $(p)$ or the electrons $(e)$.

The equations for the density $\rho_{p}=m_{p} n$ and velocity $\boldsymbol{u}_{p}$ of the ions (protons) are

$$
\begin{gathered}
\partial_{t} \rho_{p}+\nabla \cdot\left(\rho_{p} \boldsymbol{u}_{p}\right)=0 \\
\partial_{t} \boldsymbol{u}_{p}+\boldsymbol{u}_{p} \cdot \nabla \boldsymbol{u}_{p}+\frac{1}{\rho_{p}} \nabla \cdot \boldsymbol{p}_{p}-\frac{e}{m_{p}}\left(\boldsymbol{E}+\frac{1}{c} \boldsymbol{u}_{p} \wedge \boldsymbol{B}\right)=0 .
\end{gathered}
$$

Concentrating on the ion scales and thus neglecting electron inertia, the electric field $\boldsymbol{E}$ is given by Ohm's law $\boldsymbol{E}=-\frac{1}{c} \boldsymbol{u}_{p} \wedge \boldsymbol{B}+\boldsymbol{E}_{H}$, where we isolate the Hall electric field $\boldsymbol{E}_{H}=\frac{1}{n e}\left(\frac{1}{c} \boldsymbol{j} \wedge \boldsymbol{B}-\nabla \cdot \boldsymbol{p}_{e}\right)$. Here, the velocities are nonrelativistic and the electric current thus given by $\boldsymbol{j}=\frac{c}{4 \pi} \nabla \wedge \boldsymbol{B}$, with the magnetic field obeying the Faraday equation $\partial_{t} \boldsymbol{B}=-c \nabla \wedge \boldsymbol{E}$. Moreover the ion and electron pressure tensors are respectively written as $\boldsymbol{p}_{p}=\boldsymbol{p}_{p}^{G}+\Pi$ and $\boldsymbol{p}_{e}=\boldsymbol{p}_{e}^{G}$ (as the electron Larmor radius is neglected) where, for each species, the gyrotropic pressure has the form $\boldsymbol{p}_{r}^{G}=p_{\perp r} \boldsymbol{n}+p_{\| r} \tau$, with $\boldsymbol{n}=\boldsymbol{I}-\boldsymbol{b} \otimes \boldsymbol{b}$ and $\tau=\boldsymbol{b} \otimes \boldsymbol{b}$. Here, $\boldsymbol{b}=\boldsymbol{B} /|\boldsymbol{B}|$ is the unit vector along the local magnetic field.

The parallel and perpendicular pressures of each particle species obey $\left(\frac{d}{d t}=\partial_{t}+\boldsymbol{u}_{p} \cdot \nabla\right)$

$$
\begin{aligned}
& \partial_{t} p_{\| r}+\nabla \cdot\left(p_{\| r} \boldsymbol{u}_{r}\right)+\left(2 p_{\| r} \nabla \boldsymbol{u}_{r}+\nabla \cdot \boldsymbol{q}_{r}\right): \tau+\left[\left(\Pi \cdot \nabla \boldsymbol{u}_{p}\right)^{S}: \tau-\Pi: \frac{d \tau}{d t}\right] \delta_{r p}=0 \\
& \partial_{t} p_{\perp r}+\nabla \cdot\left(p_{\perp r} \boldsymbol{u}_{r}\right)+\frac{1}{2}\left(2 p_{\perp r} \nabla \boldsymbol{u}_{r}+\nabla \cdot \boldsymbol{q}_{r}\right): \boldsymbol{n}+\frac{1}{2}\left[\left(\Pi \cdot \nabla \boldsymbol{u}_{p}\right)^{S}: \boldsymbol{n}+\Pi: \frac{d \tau}{d t}\right] \delta_{r p}=0
\end{aligned}
$$

where $\boldsymbol{q}_{r}$ denotes the corresponding heat flux tensor. The electron velocity is given by $\boldsymbol{u}_{e}=\boldsymbol{u}_{p}-\boldsymbol{j} / n e$, and $\delta_{r p}$ means that the corresponding terms exist for ions only. The superscripts $S$ indicate that the corresponding tensors are symmetrized. It is in fact of interest in (2.4) to rewrite

$$
\left(\Pi \cdot \nabla \boldsymbol{u}_{p}\right)^{S}: \boldsymbol{n}=-2(\nabla \cdot \Pi) \cdot \boldsymbol{u}_{p}+2 \nabla \cdot\left(\boldsymbol{u}_{p} \cdot \Pi\right)-\left(\Pi \cdot \nabla \boldsymbol{u}_{p}\right)^{S}: \tau,
$$

in order to make more conspicuous the conservation of the energy

$$
\mathscr{E}=\frac{1}{2} \int\left(\rho_{p}\left|\boldsymbol{u}_{p}\right|^{2}+|\boldsymbol{B}|^{2}+2 p_{\perp p}+p_{\| p}+2 p_{\perp e}+p_{\| e}\right) d \boldsymbol{x} .
$$

Furthermore, the Faraday equation rewrites

$$
\partial_{t} \boldsymbol{b}+\frac{1}{|\boldsymbol{B}|}\left(\partial_{t}|\boldsymbol{B}|\right) \boldsymbol{b}=\boldsymbol{b} \cdot \nabla \boldsymbol{u}-\boldsymbol{u} \cdot \nabla \boldsymbol{b}-\frac{1}{|\boldsymbol{B}|}(\boldsymbol{u} \cdot \nabla|\boldsymbol{B}|) \boldsymbol{b}-(\nabla \cdot \boldsymbol{u}) \boldsymbol{b}-\frac{c}{|\boldsymbol{B}|} \nabla \wedge \boldsymbol{E}_{H},
$$

and, after multiplying by the tensor $\boldsymbol{n}$, one simply obtains

$$
\frac{d \boldsymbol{b}}{d t}=\left(\boldsymbol{b} \cdot \nabla \boldsymbol{u}_{p}-\frac{c}{|\boldsymbol{B}|} \nabla \wedge \boldsymbol{E}_{H}\right) \cdot \boldsymbol{n}
$$


Combining with the condition $\Pi: \tau=0$, one has

$$
\Pi: \frac{d \tau}{d t}=2 \boldsymbol{b} \cdot \Pi \cdot\left(\boldsymbol{b} \cdot \nabla \boldsymbol{u}_{p}-\frac{c}{|\boldsymbol{B}|} \nabla \wedge \boldsymbol{E}_{H}\right) .
$$

The heat flux tensor can furthermore be written $\boldsymbol{q}_{r}=\boldsymbol{S}_{r}+\sigma_{r}$, where the tensor $\sigma_{r}$ obeys the conditions $\sigma_{r}: \boldsymbol{n}=0$ and $\sigma_{r}: \tau=0$. The elements of the tensor $\boldsymbol{S}_{r}$ are classically expressed (see e.g. Goswami et al. (2005)) in terms of the components of two vectors $\boldsymbol{S}_{r}^{\|}$and $\boldsymbol{S}_{r}^{\perp}$ defined by $\boldsymbol{S}_{r}^{\|}=\boldsymbol{q}_{r}: \tau$ and $\boldsymbol{S}_{r}^{\perp}=(1 / 2) \boldsymbol{q}_{r}: \boldsymbol{n}$ that measure the directional fluxes of the parallel and perpendicular heats respectively. The usual perpendicular and parallel gyrotropic heat fluxes are given by $q_{\perp r}=\boldsymbol{S}_{r}^{\perp} \cdot \boldsymbol{b}$ and $q_{\| r}=S_{r}^{\|} \cdot \boldsymbol{b}$, and thus correspond to the fluxes along the magnetic field. We write $\boldsymbol{S}_{r}^{\perp}=q_{\perp r} \boldsymbol{b}+\boldsymbol{S}_{\perp r}^{\perp}$ and $\boldsymbol{S}_{r}^{\|}=q_{\| r} \boldsymbol{b}+\boldsymbol{S}_{\perp r}^{\|}$, and find for the $\boldsymbol{S}_{r}$ contributions to the flux terms in the gyrotropic pressure equations

$$
\begin{aligned}
& \left(\nabla \cdot \boldsymbol{S}_{r}\right): \tau=\nabla \cdot\left(q_{\| r} \boldsymbol{b}+\boldsymbol{S}_{\perp r}^{\|}\right)-2\left(q_{\perp r} \nabla \cdot \boldsymbol{b}+\boldsymbol{b} \cdot \nabla \boldsymbol{b} \cdot \boldsymbol{S}_{\perp r}^{\|}\right) \\
& \frac{1}{2}\left(\nabla \cdot \boldsymbol{S}_{r}\right): \boldsymbol{n}=\nabla \cdot\left(q_{\perp r} \boldsymbol{b}+\boldsymbol{S}_{\perp r}^{\perp}\right)+q_{\perp r} \nabla \cdot \boldsymbol{b}+\boldsymbol{b} \cdot \nabla \boldsymbol{b} \cdot \boldsymbol{S}_{\perp r}^{\|} .
\end{aligned}
$$

It also turns out that the tensor $\sigma$ does not contribute at the linear level in (2.3) and (2.4). This is shown in Passot and Sulem (2007) by substituting the kinetic expressions of the various fluid moments in the linearized equations for the perpendicular and parallel pressures, which leads to $\left(\nabla \cdot \sigma_{r}\right): \tau=0$ and $\left(\nabla \cdot \sigma_{r}\right): \boldsymbol{n}=0$. The leading order of $\sigma$ can also be evaluated in a large-scale asymptotics (see Sec. 3 ), where it is clearly seen to contribute at the nonlinear level only.

In addition to the non-gyrotropic contributions, the parallel and perpendicular heat fluxes $q_{\| r}$ and $q_{\perp r}$ must be determined either directly by closure relations, or by means of the dynamical equations provided by the fluid hierarchy, thus pushing the closure problem to the level of the gyrotropic fourth-rank moments or cumulants. In the latter case, the corresponding non-gyrotropic contributions are also to be evaluated.

\section{A large-scale fully-nonlinear FLR-Landau fluid model}

The estimate of the non-gyrotropic contribution within a large-scale asymptotics, initiated in the early sixties, is usually limited to leading order (Macmahon 1965). We shall retain this level of accuracy when dealing with the full nonlinear expressions.

\subsection{Non-gyrotropic moments}

\subsubsection{Ion non-gyrotropic pressure tensor}

Denoting by $\Omega_{p}^{L}=e B / m_{p} c$ the pointwise ion gyrofrequency, one has (see e.g. Schekochihin et al. (2010) for a simple derivation)

$$
\Pi=\frac{1}{4 \Omega_{p}^{L}}[\boldsymbol{b} \wedge \boldsymbol{W} \cdot(\boldsymbol{I}+3 \tau)-(\boldsymbol{I}+3 \tau) \cdot \boldsymbol{W} \wedge \boldsymbol{b}]+\frac{1}{\Omega_{p}^{L}}[\boldsymbol{b} \otimes(\boldsymbol{w} \wedge \boldsymbol{b})+(\boldsymbol{w} \wedge \boldsymbol{b}) \otimes \boldsymbol{b}] .
$$

Here $\boldsymbol{I}$ is the density matrix,

$$
\boldsymbol{W}=\left[p_{\perp p} \nabla \boldsymbol{u}_{p}+\nabla\left(q_{\perp p} \boldsymbol{b}\right)\right]^{S}
$$

and $\left(\partial_{\|}=\boldsymbol{b} \cdot \nabla\right)$

$$
\boldsymbol{w}=\left(p_{\perp p}-p_{\| p}\right)\left(\frac{d \boldsymbol{b}}{d t}+\partial_{\|} \boldsymbol{u}_{p}\right)+\left(3 q_{\perp p}-q_{\| p}\right) \partial_{\|} \boldsymbol{b}
$$


with $\frac{d \boldsymbol{b}}{d t}$ given by (2.8) where, at large scales, the contribution of the Hall electric field is subdominant and thus neglected, leading to

$$
\frac{d \boldsymbol{b}}{d t}+\partial_{\|} \boldsymbol{u}_{p}=2 \partial_{\|} \boldsymbol{u}_{p}-\left(\tau: \nabla \boldsymbol{u}_{p}\right) \boldsymbol{b}
$$

It follows that $\boldsymbol{w} \wedge \boldsymbol{b}$ reduces to

$$
\boldsymbol{w} \wedge \boldsymbol{b}=\left[2\left(p_{\perp p}-p_{\| p}\right) \partial_{\|} \boldsymbol{u}_{p}+\left(3 q_{\perp p}-q_{\| p}\right) \partial_{\|} \boldsymbol{b}\right] \wedge \boldsymbol{b} .
$$

\subsubsection{Non-gyrotropic heat fluxes}

Starting for example from (30) and (31) of Goswami et al. (2005), one easily establishes the expression given in Macmahon (1965) and Ramos (2005) for the vectors $S_{\perp p}^{\perp}$ and $S_{\perp p}^{\|}$measuring the transverse fluxes of perpendicular and parallel heats:

$$
\begin{aligned}
\boldsymbol{S}_{\perp p}^{\perp}= & \frac{1}{\Omega_{p}^{L}} \boldsymbol{b} \wedge\left[2 \frac{p_{\perp p}}{m_{p}} \nabla T_{\perp p}+4 q_{\perp p} \partial_{\|} \boldsymbol{u}_{p}+2 \widetilde{r}_{\| \perp} \partial_{\|} \boldsymbol{b}\right] \\
\boldsymbol{S}_{\perp p}^{\|}= & \frac{1}{\Omega_{p}^{L}} \boldsymbol{b} \wedge\left[\frac{p_{\perp p}}{m_{p}} \nabla T_{\| p}+2 \frac{T_{\| p}}{m_{p}}\left(p_{\| p}-p_{\perp p}\right) \partial_{\|} \boldsymbol{b}+2 q_{\| p} \partial_{\|} \boldsymbol{u}_{p}\right. \\
& \left.+2 q_{\perp p}\left(\boldsymbol{b} \wedge \boldsymbol{\omega}_{p}\right)+\nabla \widetilde{r}_{\| \perp p}+\left(\widetilde{r}_{\|\| p}+3 \widetilde{r}_{\| \perp p}\right) \partial_{\|} \boldsymbol{b}\right] .
\end{aligned}
$$

Here $\tilde{r}_{\| \perp p}$ and $\tilde{r}_{\|\| p}$ are gyrotropic fourth-rank cumulants. Similarly, for the electrons, the contributions relevant at ion scales read

$$
\begin{aligned}
S_{\perp e}^{\perp} & =-\frac{2 p_{\perp e}}{m_{p} \Omega_{p}^{L}} \boldsymbol{b} \wedge \nabla T_{\perp e} \\
S_{\perp e}^{\|} & =-\frac{p_{\perp e}}{m_{p} \Omega_{p}^{L}} \boldsymbol{b} \wedge\left[\nabla T_{\| e}-2 T_{\| e}\left(1-\frac{T_{\| e}}{T_{\perp e}}\right) \partial_{\|} \boldsymbol{b}\right] .
\end{aligned}
$$

As previously mentioned, the full heat flux tensor also involves the tensor $\sigma_{r}$. Following Ramos (2005), we write

$$
\begin{aligned}
\sigma_{r, i j k}= & \left(\epsilon_{i l m} \tau_{j l} n_{k n}+\epsilon_{j l m} \tau_{k l} n_{i n}+\epsilon_{k l m} \tau_{i l} n_{j n}\right. \\
& \left.+\epsilon_{i l m} \tau_{k l} n_{j n}+\epsilon_{j l m} \tau_{i l} n_{k n}+\epsilon_{k l m} \tau_{j l} n_{i n}\right) T_{r, m n}
\end{aligned}
$$

with

$$
\boldsymbol{T}_{p}=\frac{1}{4 \Omega_{p}^{L}}\left\{q_{\perp p}\left(\nabla \boldsymbol{u}_{p}\right)^{S}+\left[\frac{T_{\perp p}}{m_{p}}\left(p_{\| p}-p_{\perp p}\right)+4 r_{\| \perp p}\right](\nabla \boldsymbol{b})^{S}\right\}
$$

and

$$
\boldsymbol{T}_{e}=-\frac{1}{4 \Omega_{p}^{L}} \frac{T_{\perp e}}{m_{p}}\left(p_{\| e}-p_{\perp e}\right)(\nabla \boldsymbol{b})^{S}
$$

In (3.11),

$$
r_{\| \perp p}=\widetilde{r}_{\| \perp p}+\frac{1}{\rho_{p}} p_{\perp p} p_{\| p}
$$

is a gyrotropic fourth-rank moment.

In the equations for the gyrotropic pressures, one actually only needs $\partial_{k} \sigma_{r, i j k} \tau_{i j}=$ $-2 \sigma_{r, i j k} b_{i} \partial_{k} b_{j}$ and $\partial_{k} \sigma_{r, i j k} n_{i j}=2 \sigma_{r, i j k} b_{i} \partial_{k} b_{j}$. It follows that

$$
\partial_{k} \sigma_{r, i j k} n_{i j}=-\partial_{k} \sigma_{r, i j k} \tau_{i j}
$$


with

$$
\partial_{k} \sigma_{p, i j k} \tau_{i j}=\frac{q_{\perp p}}{2 \Omega_{p}^{L}} b_{l} \epsilon_{l k m}(\nabla \boldsymbol{b})_{k j}^{S} n_{j n}\left(\nabla \boldsymbol{u}_{p}\right)_{n m}^{S}
$$

and

$$
\partial_{k} \sigma_{e, i j k} \tau_{i j}=0
$$

making the $\sigma$-contribution relevant only for the ions.

\subsection{Closure of the fluid hierarchy}

\subsubsection{A closure for the gyrotropic heat fluxes}

In order to retain linear Landau damping, the gyrotropic heat fluxes $q_{\| r}$ and $q_{\perp r}$ can be evaluated through a so-called Landau-fluid closure, consistent with the lowfrequency large-scale linear kinetic theory. In contrast with Snyder et al. (1997) who concentrate on the purely MHD scales, we here retain leading order contributions originating from the Hall term and the FLR corrections. The derivation is made in Appendix A, starting from the formulas for the heat fluxes and the temperatures of each particle species provided by the linear kinetic theory and replacing the plasma response function by three-pole and one-pole Padé approximants respectively (Hedrick and Leboeuf 1992). Defining for each species $r$, the mean (or, in the linear theory, the equilibrium) temperatures $\bar{T}_{\perp r}=\bar{p}_{\perp r} / n_{0}$ and $\bar{T}_{\| r}=\bar{p}_{\| r} / n_{0}$, (where $n_{0}$ is the mean number density of the ions and the electrons), one introduces the instantaneous spaceaveraged thermal velocities $v_{\mathrm{th} \| \mathrm{r}}=\left(2 \bar{T}_{\| r} / m_{r}\right)^{1 / 2}$, ion gyrofrequency $\Omega_{p}=e B_{0} / m_{p} c$ and Larmor radius $r_{L}=\left(1 / \Omega_{p}\right)\left(2 \bar{T}_{\perp p} / m_{p}\right)^{1 / 2}$. One gets

$$
q_{\| r}=-\bar{p}_{\| r} v_{\mathrm{th} \| r} \frac{2}{\sqrt{\pi}} \mathrm{H} \frac{T_{\| r}}{\bar{T}_{\| r}}
$$

together with

$$
\begin{aligned}
q_{\perp p}= & -\frac{\bar{p}_{\perp p}}{\Omega_{p}} \frac{\left(\bar{T}_{\perp p}-\bar{T}_{\| p}\right)}{m_{p}} \boldsymbol{b} \cdot\left(\nabla \wedge \frac{\boldsymbol{B}}{B_{0}}\right)-\bar{p}_{\perp p} v_{\mathrm{th} \| \mathrm{p}} \\
& \times \frac{\mathrm{H}}{\sqrt{\pi}}\left[\frac{T_{\perp p}}{\bar{T}_{\perp p}}-\frac{1}{\Omega_{p}} \boldsymbol{b} \cdot\left(\nabla \wedge \boldsymbol{u}_{p}\right)+\left(\frac{\bar{T}_{\perp p}}{\bar{T}_{\| p}}-1\right) \frac{|\boldsymbol{B}|}{B_{0}}\right]
\end{aligned}
$$

and

$$
q_{\perp e}=\frac{\bar{p}_{\perp e}}{\Omega_{p}} \frac{\left(\bar{T}_{\perp e}-\bar{T}_{\| e}\right)}{m_{p}} \boldsymbol{b} \cdot\left(\nabla \wedge \frac{\boldsymbol{B}}{B_{0}}\right)-\bar{p}_{\perp e} v_{\text {th } \| \mathrm{e}} \frac{\mathrm{H}}{\sqrt{\pi}}\left[\frac{T_{\perp e}}{\bar{T}_{\perp e}}+\left(\frac{\bar{T}_{\perp e}}{\bar{T}_{\| e}}-1\right) \frac{|\boldsymbol{B}|}{B_{0}}\right] .
$$

In a purely linear analysis, $\boldsymbol{b}$ is replaced by the unit vector along the ambient magnetic field, while the operator $\mathrm{H}$, physically associated with the Landau resonance, identifies with the negative Hilbert operator in the direction of this field. In Fourier space, it then reduces to the multiplication by $i \operatorname{sgn} k_{z}$, that is to say $i k_{z} /\left|k_{z}\right|$ for $k_{z} \neq 0$ and 0 for $k_{z}=0$.

Differently, when the model is considered in the nonlinear regime, the distortion of the magnetic field lines being retained in the dynamical equations governing the gyrotropic moments, it is important to also take it into account at the level of closure (3.24)-(3.26). Otherwise, the inconsistent description of the longitudinal derivative $\partial_{\|}=\boldsymbol{b} \cdot \nabla$ in the hierarchy equations and in the linearly-evaluated terms on which it acts may lead to spurious secondary instabilities in multi-dimensional nonlinear simulations. Indeed, while terms involving the operator $\partial_{\|} \mathrm{H}$ are purely dissipative 
in the linear theory (as in Fourier space, this operator then reduces to $-\left|k_{z}\right|$ ) and also in one-space dimension (Borgogno et al. 2007; Laveder et al. 2013), in several dimensions a mismatch in the description of the two operators may produce a small antidiffusive contribution (Passot et al. 2014). In the present context, the $\beta$ of the plasma is not assumed to be small, making the field line distortion not fully negligible. Furthermore, the $\mathrm{H}$ operator also arises in the non-gyrotropic pressure tensor when closing the hierarchy at the level of the heat fluxes. We are thus led to use a different modeling. As accurately retaining the magnetic field distortion requires the replacement of the Hilbert transform along the ambient field by an integral along the individual magnetic field lines, which is hardly feasible on the present day computers, we resorted to provide a semi-phenomenological modeling of the $\mathrm{H}$ operator. While the $i k_{z}$ factor arising in the numerator of the Fourier representation of the linearized operator is easily replaced by the directional derivative $\partial_{\|}$in physical space, finding an equivalent of the denominator $\left|k_{z}\right|$ is less straightforward. In the model used by Sharma et al. (2006), the Fourier multiplier $\left|k_{z}\right|^{-1}$ is simply replaced by a typical scale, which thus transforms $\partial_{\|} \mathrm{H}$ into a diffusive operator along the field lines, as in a weakly collisional regime. The non-local character of the Landau operator associated with the fact that $\mathrm{H}$ is a zero-order operator, seems nevertheless an important feature that we choose to preserve. The simple approach is to keep the Fourier multiplier $\left|k_{z}\right|^{-1}$ as is, with the shortcoming that it displays a singularity for all the modes with wavevector purely transverse to the ambient field. This suggests to replace $\left|k_{z}\right|^{-1}$ by $\left(\left|k_{z}\right|+c\right)^{-1}$ where $c$ is a small constant, relevant only for the Fourier modes with $k_{z}=0$. Such modes are not affected within a purely linear description while, in the case of finite locally uniform oblique field line perturbations, they are here subject to a diffusive decay along the transverse directions. Another possibility is to replace $\mathrm{H}$ by $\left[(\boldsymbol{k} \cdot \bar{\tau} \cdot \boldsymbol{k})^{-\frac{1}{2}}\right] \partial_{\|}$(here and below the hat indicates the inverse Fourier transform) where, in order to keep the operator local in Fourier space, the expected tensor $\tau$ has been replaced by its instantaneous space average $\bar{\tau}$. With such a formulation, the operator is generically not singular on the numerical grid. It also provides a linearly exact approximation whatever the direction of the ambient magnetic field (up to the use of Padé approximants), and thus requires that the distortion of the magnetic field lines be moderate. Optimizing the modeling of the Landau operator is an important issue that deserves further investigations.

Note that equation (3.18) for the ion perpendicular heat flux identifies with the large-scale limit of the general expression derived in Passot and Sulem (2007) by expressing $q_{\perp p}$ by means of the dynamical equation for $T_{\perp p}$ and designing a closure formula from the kinetic expression of the latter quantity.

Furthermore, the ion non-gyrotropic heat-flux vectors $S_{\perp p}^{\perp}$ and $S_{\perp p}^{\|}$, together with the tensor $\boldsymbol{T}_{p}$, given in the previous section, involve the gyrotropic fourth-rank cumulants $\widetilde{r}_{\|\| p}$ and $\widetilde{r}_{\| \perp p}$. These latter quantities are evaluated on the basis of the linear kinetic theory in (11) and (12) of Passot et al. (2012). Their large-scale limits, we are here interested in, are explicitly given in the next section. The gyrotropic perpendicular heat flux $q_{\perp p}$ enters these expressions. When substituting from (3.18), one gets

$$
\begin{aligned}
& \widetilde{r}_{\|\| p}=-\frac{3}{2} n_{0} v_{\mathrm{th} \| r}^{2} T_{\| p}^{\prime} \\
& \widetilde{r}_{\| \perp p}=-\frac{\bar{p}_{\perp p}}{2} v_{\mathrm{th} \| r}^{2}\left[\frac{T_{\perp p}^{\prime}}{\bar{T}_{\perp p}}-\frac{1}{\Omega_{p}} \boldsymbol{b} \cdot\left(\nabla \wedge \boldsymbol{u}_{p}\right)+\left(\frac{\bar{T}_{\perp p}}{\bar{T}_{\| p}}-1\right) \frac{|\boldsymbol{B}|}{B_{0}}\right] .
\end{aligned}
$$

where prime refers to fluctuations about the instantaneous spatial average. 


\subsubsection{Advantage of a higher order closure?}

A natural question is whether it is worthwhile to push the fluid hierarchy to higher order by keeping the dynamical equations for the gyrotropic heat fluxes $q_{\| r}$ and $q_{\perp r}$ and closing the fluid hierarchy at the level of the fourth-rank moments. The full nonlinear equations are given in Ramos (2005) and turn out to be rather intricate. In order to build a model with a reasonable degree of complexity, it appears suitable to simplify these equations. As the fourth-rank velocity cumulants are evaluated in the framework of the linear kinetic theory, it seems appropriate to prescribe that, in the equations for the gyrotropic heat fluxes, the terms involving non-gyrotropic contributions are retained only if they survive when the equations are linearized about a uniform equilibrium. The equations for the gyrotropic heat fluxes then reduce to $\left(\rho_{e} / \rho_{p}=m_{e} / m_{p}\right)$

$$
\begin{aligned}
& \partial_{t} q_{\| r}+\nabla \cdot\left(q_{\| r} \boldsymbol{u}_{r}\right)+3 q_{\| r} \nabla \boldsymbol{u}_{r}: \tau+3 p_{\| r} \partial_{\|}\left(\frac{p_{\| r}}{\rho_{r}}\right)+\nabla \cdot\left(\widetilde{r}_{\|\| r} \boldsymbol{b}\right) \\
& \quad-3 \widetilde{r}_{\| \perp r} \nabla \cdot \boldsymbol{b}+R_{\| r}^{N G}=0 \\
& \partial_{t} q_{\perp r}+\nabla \cdot\left(\boldsymbol{u}_{r} q_{\perp r}\right)+q_{\perp r} \nabla \cdot \boldsymbol{u}_{r}+p_{\| r} \partial_{\|}\left(\frac{p_{\perp r}}{\rho_{r}}\right)+\nabla \cdot\left(\widetilde{r}_{\| \perp r} \boldsymbol{b}\right) \\
& \quad+\left[\left(p_{\| r}-p_{\perp r}\right) \frac{p_{\perp r}}{\rho_{r}}-\widetilde{r}_{\perp \perp r}+\widetilde{r}_{\| \perp r}\right] \nabla \cdot \boldsymbol{b}+\frac{p_{\perp p}}{\rho_{p}} \nabla \cdot(\boldsymbol{n} \cdot \Pi) \delta_{r p}+R_{\perp r}^{N G}=0,
\end{aligned}
$$

where the gyrotropic fourth-rank cumulants $\widetilde{r}_{\|\| r}, \widetilde{r}_{\| \perp r}$ and $\widetilde{r}_{\perp \perp r}$ and the contributions $R_{\| r}^{N G}$ and $R_{\perp r}^{N G}$ originating from the non-gyrotropic fourth-rank cumulants are to be evaluated to close the hierarchy. Note that $\nabla \cdot \boldsymbol{b}=-(1 /|\boldsymbol{B}|) \partial_{\|}|\boldsymbol{B}|=-\partial_{\|} \ln (|\boldsymbol{B}|)$.

When, starting from the predictions of the low-frequency linear theory, the gyrotropic fourth-rank cumulants $\widetilde{r}_{\|\| r}$ and $\widetilde{r}_{\| \perp r}$ are evaluated by using four-pole and two-pole Padé approximants respectively for the plasma response function (Hedrick and Leboeuf 1992), one gets the closure relations provided by (11)-(15) of Passot et al. (2012) which, when keeping only the first-order corrections in a large-scale expansion, reduce to

$$
\begin{aligned}
& \widetilde{r}_{\|\| r}=\frac{32-9 \pi}{2(3 \pi-8)} n_{0} v_{\mathrm{th} \| r}^{2} T_{\| r}^{\prime}-\frac{2 \sqrt{\pi}}{3 \pi-8} v_{\mathrm{th} \| r} \mathrm{H} q_{\| r} \\
& \widetilde{r}_{\| \perp p}=-\frac{\sqrt{\pi}}{2} v_{\mathrm{th} \| p} \mathrm{H}\left[q_{\perp p}+\frac{v_{\mathrm{th} \| p}^{2}}{2 \Omega_{p}} \frac{\bar{T}_{\perp p}}{\bar{T}_{\| p}}\left(\bar{p}_{\perp p}-\bar{p}_{\| p}\right)\left(\nabla \wedge \frac{\boldsymbol{B}}{B_{0}}\right) \cdot \boldsymbol{b}\right] \\
& \widetilde{r}_{\| \perp e}=-\frac{\sqrt{\pi}}{2} v_{\mathrm{th} \| e} \mathrm{H}\left[q_{\perp e}-\frac{v_{\mathrm{th} \| p}^{2}}{2 \Omega_{p}} \frac{\bar{T}_{\perp e}}{\bar{T}_{\| p}}\left(\bar{p}_{\perp e}-\bar{p}_{\| e}\right)\left(\nabla \wedge \frac{\boldsymbol{B}}{B_{0}}\right) \cdot \boldsymbol{b}\right] \\
& \widetilde{r}_{\perp \perp r}=0 .
\end{aligned}
$$

With the same ordering, one has

$$
\frac{p_{\perp p}}{\rho_{p}} \nabla \cdot(\boldsymbol{n} \cdot \Pi)+R_{\perp p}^{N G}=-\frac{\bar{T}_{\perp p}}{m_{p} \Omega_{p}}\left(\bar{p}_{\perp p}-2 \bar{p}_{\| p}\right) \partial_{\|}\left(\nabla \cdot \frac{c \boldsymbol{E}}{B_{0}}\right)
$$

and

$$
R_{\perp e}^{N G}=\frac{\bar{T}_{\perp e}}{m_{p} \Omega_{p}}\left(\bar{p}_{\perp e}-2 \bar{p}_{\| e}\right) \partial_{\|}\left(\nabla \cdot \frac{c \boldsymbol{E}}{B_{0}}\right)
$$

where, for consistency, we use the further approximation $c \boldsymbol{E} / B_{0}=\boldsymbol{b} \wedge \boldsymbol{u}_{p}$. As the present estimates are based on the linear kinetic theory only, one can in fact simply 
write

$$
\nabla \cdot \frac{c \boldsymbol{E}}{B_{0}}=-\boldsymbol{b} \cdot\left(\nabla \wedge \boldsymbol{u}_{p}\right)
$$

\section{Validation and limits of the models}

\subsection{Dispersion and decay of kinetic Alfvén waves}

In order to validate the above models, we analyze in this section their predictions concerning the dispersion and damping of kinetic Alfvén waves. Alfvénic fluctuations are indeed a main component of Solar Wind turbulence, and a correct description of their dissipation is most useful in modeling such a medium. The case of a wave propagating in a direction quasi-transverse to the ambient magnetic field is considered in Fig. 1 where the propagation angle $\theta=89.94^{\circ}$ is chosen in order push the ion-cyclotron resonance at very small scales. Isotropic and equal ion and electron temperatures are considered for different values of the beta parameter, namely $\beta=0.1$ (top), 1 (middle) and 10 (bottom). For the two models discussed above, we globally observe an excellent agreement with the prediction of the kinetic theory obtained with the WHAMP software (Rönnmark 1982), for both the real frequency (left) and the decay rate (right), even at scales significantly smaller than those for which the asymptotics is supposed to be valid. It is nevertheless noticeable that for $\beta=0.1$, the damping resulting from the heat flux closure displays a significantly better behavior a small scales $k_{\perp} r_{L}>10$ than the fourth-rank moment closure.

A comparable accuracy of both closures still holds when decreasing the angle to $\theta=85^{\circ}$, although on a less extended spectral range (Fig. 2).

A ion temperature anisotropy $a_{p}=T_{\perp p} / T_{\| p}=1.5$ is assumed in Fig. 3 where $\beta_{\| p}=1, \theta=89.94^{\circ}$ and $T_{\perp e}=T_{\| e}=T_{\| p}$. In this case, the closure at the level of the fourth-rank cumulants appears to be more accurate, especially at small scales. Several comments are in order. First, in all the considered examples the damping rate remains accurate at smaller scales than the real frequency, ensuring in particular a precise description of the dissipation at scales comparable or even smaller than the ion Larmor radius. Furthermore, retaining the $O\left(\Omega_{p}^{-1}\right)$ corrections in the kinetic estimate of the gyrotropic heat fluxes when the closure is performed at this level, or in the non-gyrotropic contributions to the fourth-rank cumulants when dynamical equations are retained for the parallel and perpendicular heat fluxes, is a main requirement to prevent the development of spurious linear instabilities. It is important to note that in the former closure, the first-order correction to the perpendicular heat flux, although formally subdominant, is to be retained even when substituted in the non-gyrotropic pressure tensor, as this contribution involves the parallel vorticity and current, which are dominant components of the kinetic Alfvén eigenmodes.

The above analysis thus leads to the conclusion that, for weak temperature anisotropy, the heat flux closure provides a satisfactory modeling, with no need to push the fluid hierarchy to higher order.

\subsection{Mirror instability}

When the temperature anisotropy $a_{p}=T_{\perp p} / T_{\| p}$ is increased beyond a critical value depending on the plasma beta, mirror modes become unstable. This micro-instability which is driven by the Landau resonance and arrested at small scales by finite Lamor radius effects, is supposed to play an important role in monitoring the temperature anisotropy in the Solar Wind. Observations indeed suggest that it constrains the system to remain close to threshold (Matteini et al. 2012, and references therein), a 

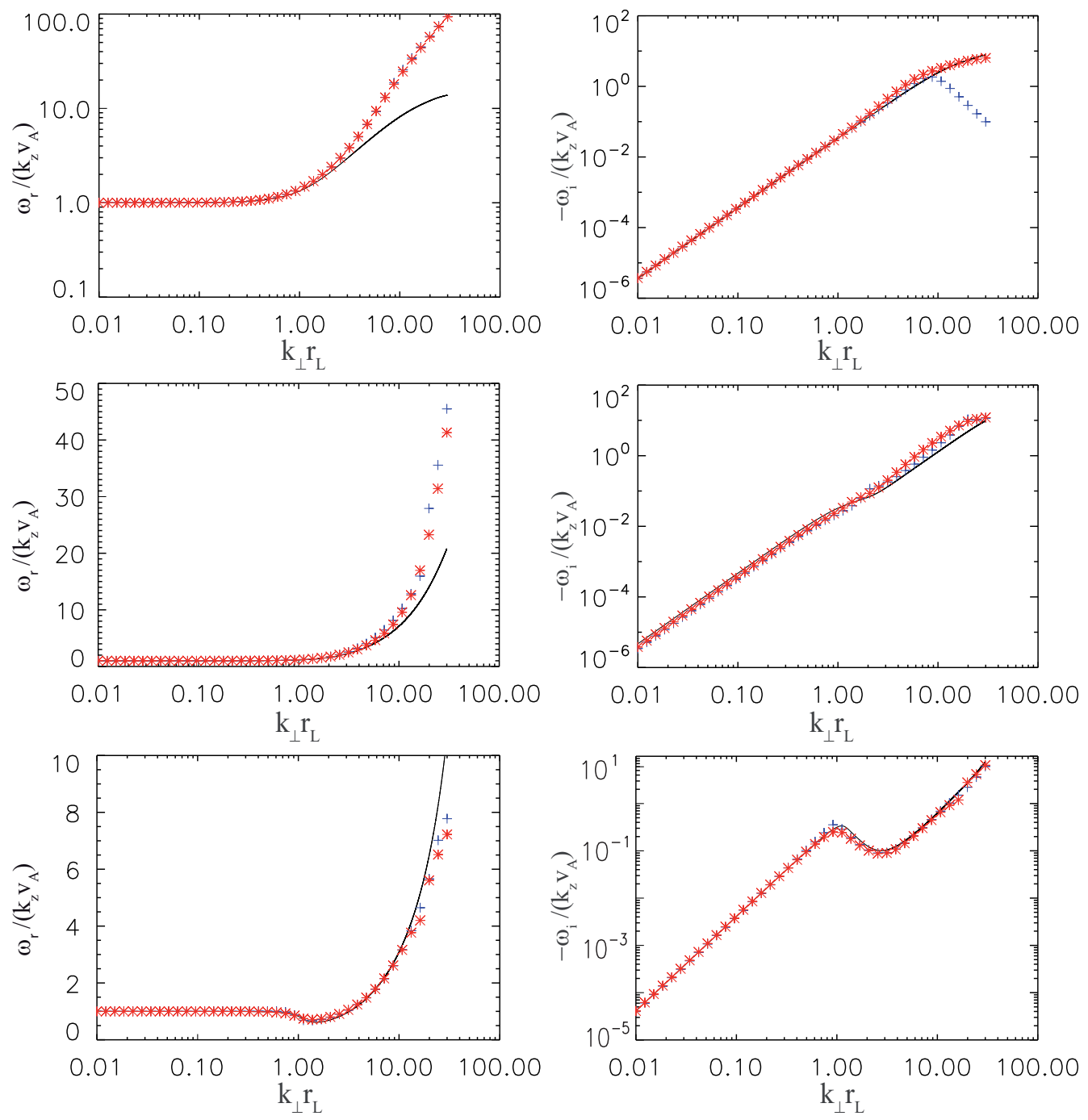

FIGURE 1. Frequency $\omega_{r}$ and damping rate $-\omega_{i}$ normalized by $k_{z} v_{A}$ (where $k_{z}=k \cos \theta$ and $v_{A}=B_{0} / \sqrt{4 \pi \rho_{0}}$ is the Alfvén velocity) of a kinetic Alfvén wave propagating in a direction making an angle $\theta=89.94^{\circ}$, versus $k_{\perp} r_{L}$, for equal and isotropic ion and electron temperatures, and $\beta=0.1$ (top), 1 (middle), 10 (bottom). The solid line corresponds to the full kinetic theory using the WHAMP software, while red stars and blue crosses result from the model equations with the closure performed at the level of the heat-fluxes and of the fourth-rank moments respectively. In some cases, the blue crosses are not visible, the predictions of the two models being very close.

conclusion also supported by numerical simulations of a one-dimensional reduction of the model described in Sec. 5.1 (Laveder et al. 2011).

The mirror instability growth rates predicted by the two large-scale models described in Sec. 3 are displayed in Fig. 4 (parameters given in the caption) in regimes close to threshold (left) and relatively far from it (right), together with the result of the FLR-Landau fluid model (Passot and Sulem 2007; Passot et al. 2012), discussed in Sec. 5.1, which retains an accurate description of the linear kinetic properties at the quasi-transverse sub-ion scales. We observe that near threshold both the large-scale 

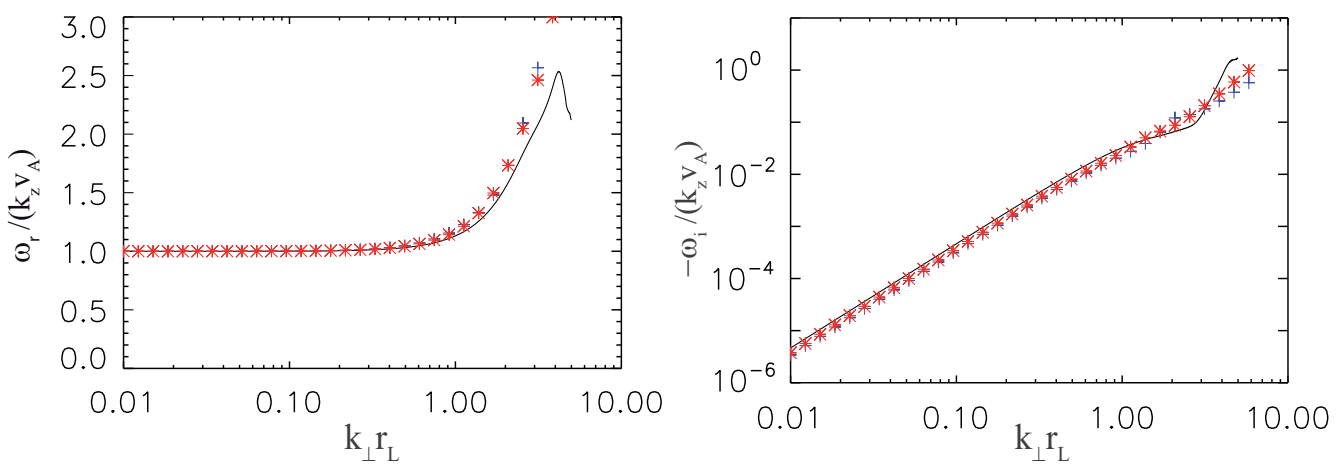

Figure 2. Same as Fig. 1 for $\theta=85^{\circ}$ and $\beta_{\| p}=1$.
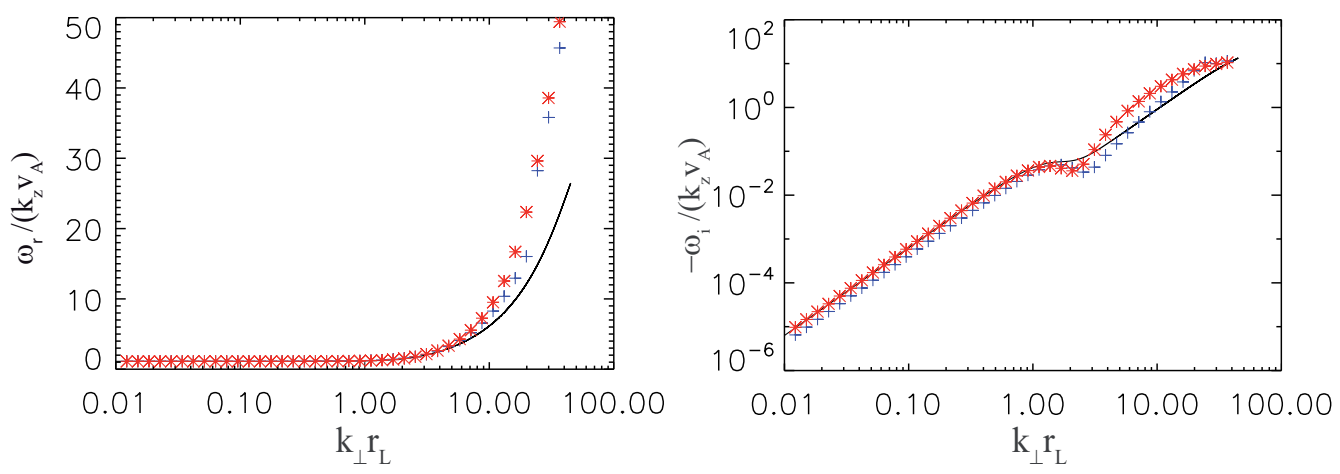

Figure 3. Same as Fig. 1 for $\theta=89.94^{\circ}$, proton temperature anisotropy $a_{p}=T_{\perp p} / T_{\| p}=1.5$, $T_{\perp e}=T_{\| e}=T_{\| i}$, and $\beta_{\| p}=1$.
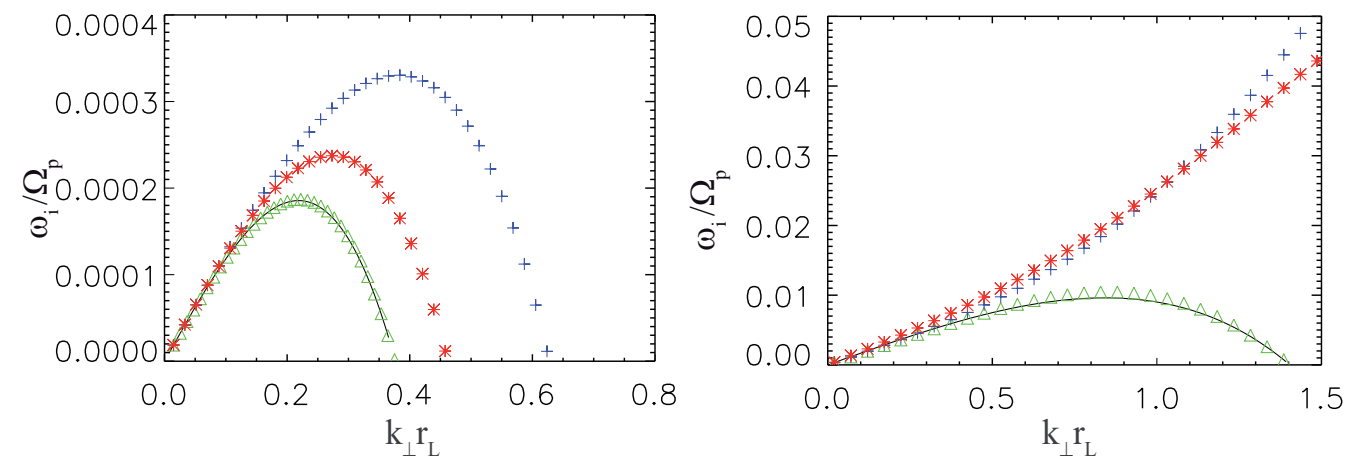

FiguRE 4. Mirror instability growth rate $\omega_{i}$ normalized by the ion gyrofrequency $\Omega_{p}$, versus $k_{\perp} r_{L}$, for a propagation angle $\theta=85^{\circ}$, in conditions near (left) and far (right) from threshold. In the former regime, $T_{\perp p} / T_{\| p}=1.45, T_{\perp e} / T_{\| e}=1.1, T_{\| e}=T_{\| p}$ and $\beta_{\| p}=1.5$. In the latter, $T_{\perp p} / T_{\| p}=2, T_{\perp e} / T_{\| e}=1, T_{\| e}=T_{\| p}$ and $\beta_{\| p}=2$. As in the previous figures, the solid line refers to the predictions of the full kinetic theory using the WHAMP software, while the red stars and the blue crosses are associated with the heat-flux and fourth-rank moment large-scale closures respectively. The green triangles correspond to the FLR-Landau fluid model described in Sec. 5.1. 


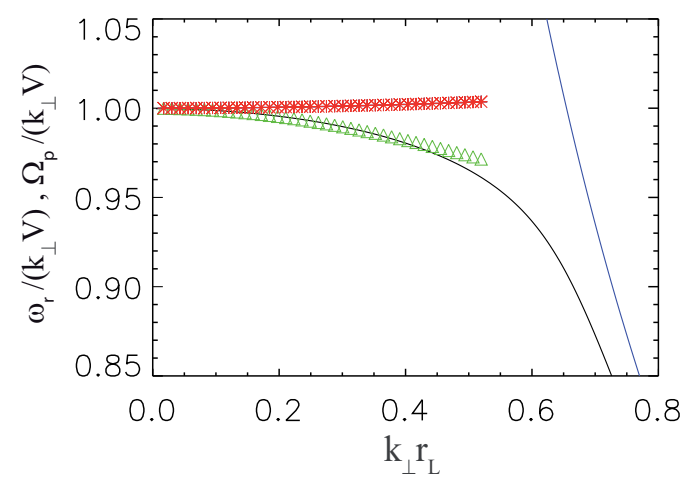

FIGURE 5. Frequency $\omega_{r}$, normalized by $k_{\perp} V$ (where $V=v_{A}\left(1+\beta_{T}\right)^{1 / 2}$ and $\beta_{T}=\beta_{p}+\beta_{e}$ ) of a purely transverse fast magnetosonic wave, versus $k_{\perp} r_{L}$, for equal and isotropic ion and electron temperatures and $\beta_{p}=\beta_{e}=3$ : red stars and blue crosses (almost exactly superimposed) correspond to the two large-scale models and green triangles to the FLR-Landau fluid discussed in Sec. 5, while the black solid line refers to the full kinetic theory and the blue solid line to the normalized cyclotron frequency $\Omega_{p} /\left(k_{\perp} V\right)$.

models are quantitatively accurate at very large scale only, but already display a significant deviation from the kinetic theory at $k_{\perp} r_{L}=0.2$, the error being in fact larger in the case of the fourth-rank moment closure. Far from threshold, both large-scale models are incorrect even qualitatively, as no arrest of the instability is obtained at small scales. In contrast, in both regimes, the growth rate predicted by the kinetic theory is accurately reproduced by the FLR-Landau fluid model discussed in Sec. 5.

\subsection{Fast magnetosonic waves}

As discussed for example in Passot et al. (2012), retaining only the leading-order FLR corrections is not sufficient to accurately capture the dispersion of fast magnetosonic waves, even at very large scales. This point is illustrated in Fig. 5 in the case of purely transverse propagation, in a plasma with equal and isotropic ion and electron temperatures and $\beta_{p}=\beta_{e}=3$. The real frequency $\omega_{r}$ (no damping is present in the transverse direction), normalized by $k_{\perp} V$ is plotted versus $k_{\perp} r_{L}$. Here, $V=v_{A}\left(1+\beta_{T}\right)^{1 / 2}$, where $v_{A}=B_{0} / \sqrt{4 \pi \rho_{0}}$ denotes the Alfvén velocity and $\beta_{T}=\beta_{p}+\beta_{e}$. We note that the sign of the dispersion is in particular not reproduced. Differently, a rather satisfactory agreement is obtained with the FLR-Landau fluid at large enough scales (typically $k_{\perp} r_{L}<0.4$ ), the discrepancy with the kinetic theory visible at smaller scales originating from the effect of the ion-cyclotron resonance. We remind that such fast waves are averaged out within the gyrokinetic formalism. We indeed see in Fig. 5 that the (normalized) frequency obtained from the full kinetic theory (black solid line) tends to approach and asymptotes the cyclotron frequency (blue solid line).

\section{Beyond large-scale modeling}

As discussed in Sec. 4.2, a large-scale expansion of the non-gyrotropic moments does not permit a satisfactory description of the mirror instability which is in contrast well reproduced by the FLR-Landau fluid model (Passot and Sulem 2007; Passot 
et al. 2012). Based on the linear kinetic theory within the gyrokinetic ordering, this approach is indeed designed for simulating the ion scales. It however does not retain the nonlinearities associated with the FLR corrections that are evaluated at the linear level only. This suggests to construct a new model where a linearly accurate description of the small-scale FLR corrections is supplemented to the large-scale nonlinear model. For this purpose, it is convenient to first briefly revisit the FLR-Landau fluid.

\subsection{A FLR-Landau fluid model for the sub-ion scales}

While the models described in Sec. 3 involve a fully non-linear description of FLR corrections within a large-scale perturbative expansion, a different strategy is needed in order to accurately simulate the sub-ion scale. In this context, the approach developed in Passot and Sulem (2007) and Passot et al. (2012) consists in determining the non-gyrotropic moments from the low-frequency linear kinetic theory. In its original form, the resulting closed system is suitable to address the linear dynamics and indeed successfully predicts the dispersion and damping of the kinetic Alfvén waves, together with their polarization and magnetic compressibility (Hunana et al. $2013 \mathrm{a}, \mathrm{b})$. As already mentioned, this model also accurately reproduces the mirror instability and its quenching at small scales. Nevertheless, in the multidimensional nonlinear regime, the distortion of the magnetic field lines has to be taken into account when evaluating the kinetic contributions, and this even though accuracy is ensured at the linear level only. Otherwise, when plugging the kinetic contributions within the nonlinear dynamical equations (which themselves involve derivatives along the magnetic field lines), the mismatch between the two descriptions may induce spurious secondary instabilities associated with the presence of small anti-diffusive terms (Passot et al. 2014).

This model is closed at the level of the fourth-rank cumulants, and thus involves heat flux equations, taken in the form of equations (3.22) and (3.23) which only involve the non-gyrotropic quantities contributing to the linear theory. In the same spirit, a simplification can be made in the pressure equations by dropping the (nonlinear) contributions of the tensors $\sigma_{p}$ and $\Pi$. In order to preserve energy conservation, the subdominant term $-2(\nabla \cdot \Pi) \cdot \boldsymbol{u}_{p}$ can nevertheless be retained in the equation for the ion perpendicular pressure. In this framework, only the quantities $\nabla \cdot \Pi_{\perp}, \nabla \cdot S_{\perp r}^{\perp}$ and $\nabla \cdot S_{\perp r}^{\|}$are to be estimated. Retaining the work of the non-gyrotropic pressure forces in the equations for the gyrotropic pressures, which was shown to contribute in some instances to the heating of the plasma (Laveder et al. 2013), in contrast requires estimating the full tensor $\Pi$ together with the vectors $S_{\perp r}^{\perp}$ and $S_{\perp \|_{r}}$.

\subsubsection{Fourth-rank cumulants}

When the Landau fluid closure is extended to small scales, the fourth-rank cumulants are given by

$$
\begin{aligned}
& \widetilde{r}_{\|\| r}=\frac{32-9 \pi}{2(3 \pi-8)} n_{0} v_{\mathrm{th} \| r}^{2} T_{\| r}^{\prime}-\frac{2 \sqrt{\pi}}{3 \pi-8} v_{\mathrm{th} \| r} \mathrm{H} q_{\| r} \\
& \widetilde{r}_{\| \perp p}=-\frac{\sqrt{\pi}}{2} v_{\mathrm{th} \| p} \mathrm{H}\left[q_{\perp p}+\frac{v_{\mathrm{th} \| p}^{2}}{2 \Omega_{p}} \frac{\bar{T}_{\perp p}}{\bar{T}_{\| p}}\left(\bar{p}_{\perp p}-\bar{p}_{\| p}\right) \widehat{\mathfrak{R}_{\| \perp}}\left(\nabla \wedge \frac{\boldsymbol{B}}{B_{0}}\right) \cdot \boldsymbol{b}\right] \\
& \widetilde{r}_{\| \perp e}=-\frac{\sqrt{\pi}}{2} v_{\mathrm{th} \| e} \mathrm{H}\left[q_{\perp e}-\frac{v_{\mathrm{th} \| p}^{2}}{2 \Omega_{p}} \frac{\bar{T}_{\perp e}}{\bar{T}_{\| p}}\left(\bar{p}_{\perp e}-\bar{p}_{\| e}\right)\left(\nabla \wedge \frac{\boldsymbol{B}}{B_{0}}\right) \cdot \boldsymbol{b}\right]
\end{aligned}
$$




$$
\begin{aligned}
& \widetilde{r}_{\perp \perp p}=\bar{p}_{\perp p} \frac{\bar{T}_{\perp p}}{m_{p}}\left[\widehat{\mathfrak{R}_{\perp}^{[1]}} \frac{\rho}{\rho_{0}}+\frac{1}{\Omega_{p}} \widehat{\mathfrak{R}_{\perp}^{[2]}}\left(\nabla \wedge \boldsymbol{u}_{p}\right) \cdot \boldsymbol{b}+\widehat{\mathfrak{R}_{\perp}^{[3]}} \frac{T_{\perp p}}{\bar{T}_{\perp p}}\right] \\
& \widetilde{r}_{\perp \perp e}=0 .
\end{aligned}
$$

The widehat symbols refer to integral operators obtained by inverse Fourier transform, with respect of the transverse vector $\boldsymbol{k}_{\perp}$, of functions indicated by Gothic script and that we refer to as 'Bessel coefficients'. They are explicit functions of the nondimensional square wavenumber $b=k_{\perp}^{2} r_{L}^{2} / 2=k_{\perp}^{2} T_{\perp p} /\left(m_{p} \Omega_{p}^{2}\right)$, built from the functions $\Gamma_{0}(b)$ and $\Gamma_{1}(b)$ defined by $\Gamma_{\nu}(b)=e^{-b} I_{\nu}(b)$ where $I_{v}$ is the modified Bessel function of first kind. Bessel coefficients are listed in Table 1, together with their asymptotic behavior in the large-scale limit $b \rightarrow 0$, based on the expansion $\Gamma_{0}(b)=1-b+\frac{3}{4} b^{2}-\frac{5}{12} b^{3}+O\left(b^{4}\right)$ and $\Gamma_{1}(b)=\frac{1}{2} b-\frac{1}{2} b^{2}+\frac{5}{16} b^{3}+O\left(b^{4}\right)$. For consistency with the modeling of the $\mathrm{H}$ operator, the square wavenumber $k_{\perp}^{2}$ is replaced by $\boldsymbol{k} \cdot \overline{\boldsymbol{n}} \cdot \boldsymbol{k}$ in the definition of the variable $b$ that enters the Bessel coefficients. Using such a closure in the nonlinear context requires a moderate distortion of the magnetic field lines.

\subsubsection{Non-gyrotropic pressure tensor}

The divergence of the non-gyrotropic pressure tensor, whose components are defined by $(\nabla \cdot \Pi)_{i}=\partial_{j} \Pi_{i j}$, is written

$$
\nabla \cdot \Pi=\nabla \cdot\left[-\mathscr{A} \boldsymbol{n}+\mathscr{B} \epsilon \cdot \boldsymbol{b}+\boldsymbol{b} \otimes \boldsymbol{\Pi}_{\|}+\boldsymbol{\Pi}_{\|} \otimes \boldsymbol{b}\right],
$$

where the tensor within the square brackets can a priori differ from $\Pi$ by a zero-divergence contribution. Here, the additional contribution $-\left(\boldsymbol{\Pi}_{\|} \cdot \boldsymbol{b}\right) \tau$, a priori present within the square brackets in order to prevent double counting, actually vanishes. Furthermore, $\epsilon$ denotes the fundamental antisymetric third-rank tensor. The expression of $\nabla \cdot \Pi$ given by equation (5.6) differs from that directly obtained from the linear kinetic theory in Passot and Sulem (2007) and Passot et al. (2012), by the replacement of the unit vector along the ambient field by the unit vector in the direction of the local magnetic field. This transformation of the purely linear expressions derived from the kinetic theory into nonlinear formulas involving the local magnetic field, implies some arbitrariness. Inspection of (3.1)-(3.5) for the largescale FLR contributions nevertheless reveals that it is suitable to isolate the Hall contribution $\boldsymbol{E}_{H}$ (that scales like $1 / \Omega_{p}$ ) to the electric field and then to replace $\frac{c}{B_{0}} \boldsymbol{n}: \nabla \boldsymbol{E}$ by $-\boldsymbol{b} \cdot\left(\nabla \wedge \boldsymbol{u}_{p}\right)+\frac{c}{B_{0}} \boldsymbol{n}: \nabla \boldsymbol{E}_{H}$, and $\nabla \wedge \frac{c \boldsymbol{E}}{B_{0}} \cdot \boldsymbol{b}$ by $\boldsymbol{n}: \nabla \boldsymbol{u}_{p}+\boldsymbol{b} \cdot\left(\nabla \wedge \frac{c \boldsymbol{E}_{H}}{B_{0}}\right)$, thus dropping subdominant nonlinear terms proportional to magnetic field derivatives. The potentials $\mathscr{A}$ and $\mathscr{B}$ then rewrite

$$
\begin{aligned}
& \mathscr{A}=\bar{p}_{\perp p}\left[\frac{1}{\Omega_{p}} \widehat{\mathfrak{A}_{1}} \boldsymbol{b} \cdot\left(\nabla \wedge \boldsymbol{u}_{p}\right)+\widehat{\mathfrak{A}}_{2} \frac{T_{\perp p}}{\bar{T}_{\perp p}}\right] \\
& \mathscr{B}=\frac{\bar{p}_{\perp p}}{\Omega_{p}}\left[\widehat{\mathfrak{B}}_{3} \boldsymbol{n}: \nabla \boldsymbol{u}_{p}-\widehat{\mathfrak{B}}_{1} \boldsymbol{b} \cdot\left(\nabla \wedge \frac{c \boldsymbol{E}_{H}}{B_{0}}\right)\right] .
\end{aligned}
$$

Furthermore, we write

$$
\boldsymbol{\Pi}_{\|}=\frac{1}{\Omega_{p}}\left(2 \bar{p}_{\| p}-\bar{p}_{\perp p}\right)\left(\boldsymbol{b} \wedge \partial_{\|} \boldsymbol{u}+\boldsymbol{n} \cdot \partial_{\|} \frac{c \boldsymbol{E}_{H}}{B_{0}}\right)-\boldsymbol{n} \cdot \nabla \mathscr{C}_{2}-\boldsymbol{b} \wedge \nabla \mathscr{D}_{2},
$$

which implies $\boldsymbol{\Pi}_{\|} \cdot \boldsymbol{b}=0$, as previously mentioned. In (5.9), the first term originates from the combination of contributions from the original potentials $\mathscr{C}$ and $\mathscr{D}$ introduced in Passot et al. (2012) that are individually singular in the transverse spectral plane. The non singular contributions $\mathscr{C}_{2}$ and $\mathscr{D}_{2}$ to these potentials take the 
Defining:

$$
\begin{aligned}
& \Lambda_{1}=\Gamma_{0}(b)-\Gamma_{1}(b) \\
& \Lambda_{2}=b \Lambda_{1} \\
& \Lambda_{3}=1-\Gamma_{0}(b)
\end{aligned}
$$

Bessel coefficients

$$
\begin{aligned}
& \mathfrak{A}_{1}=1-\Gamma_{1} / \Lambda_{2}-\mathfrak{A}_{2} \\
& \mathfrak{A}_{2}=-\Gamma_{1} / \Gamma_{0} \\
& \mathfrak{B}_{1}=\Lambda_{1}\left(\Lambda_{1} / \Lambda_{3}+2\right)-1 / b \\
& \mathfrak{B}_{2}=\mathfrak{E}_{3} / b \\
& \mathfrak{B}_{3}=\mathfrak{B}_{2}-\mathfrak{B}_{1} \\
& \mathfrak{C}_{1}=\Lambda_{1}-1 \\
& \mathfrak{C}_{2}=\Lambda_{3} / b \\
& \mathfrak{C}_{3}=\mathfrak{C}_{1} / b \\
& \mathfrak{C}_{4}=\left(\mathfrak{C}_{2}-1\right) / b \\
& \mathfrak{D}_{1}=\Lambda_{1} /\left(\Gamma_{0}-\Gamma_{1} / 2-\Lambda_{2}\right) \\
& \mathfrak{D}_{2}=1-\Lambda_{1}-\mathfrak{D}_{1}\left(\Lambda_{2}+\Lambda_{3}\right) \\
& \mathfrak{D}_{3}=\mathfrak{D}_{1} /\left(1+b \mathfrak{D}_{1}\right) \\
& \mathfrak{D}_{4}=\mathfrak{D}_{2} /\left(1+b \mathfrak{D}_{1}\right) \\
& \mathfrak{D}_{5}=\mathfrak{D}_{4} / b \\
& \mathfrak{D}_{6}=\mathfrak{D}_{3} \Gamma_{1} \\
& \mathfrak{E}_{1}=-\Lambda_{1} \mathfrak{E}_{3}+2 \Lambda_{2}-\Gamma_{1} \\
& \mathfrak{E}_{3}=-1+\Lambda_{2} / \Lambda_{3} \\
& \mathfrak{E}_{4}=\mathfrak{E}_{1} / b \\
& \mathfrak{E}_{5}=\mathfrak{E}_{3} / b \\
& \mathfrak{E}_{6}=\mathfrak{E}_{4}-\mathfrak{E}_{5} \\
& \mathfrak{F}_{1}=\Gamma_{1} / \Lambda_{2}+2 \mathfrak{A}_{2} / b \\
& \mathfrak{G}_{2}=\left(\mathfrak{C}_{2}-1\right) / b \\
& \mathfrak{H}_{1}=-2 b+\Gamma_{1} / \Lambda_{1} \\
& \mathfrak{H}_{2}=\Gamma_{0} \mathfrak{H}_{1}+b \Lambda_{1}-\Gamma_{1} \\
& \left.\mathfrak{H}_{3}=\Gamma_{1}-2\right) / \Gamma_{0}-1 \\
& \mathfrak{H}_{4}=-\Lambda_{3} / \Lambda_{2}+\mathfrak{H}_{3} \\
& \mathfrak{R}_{\| \perp}=\Lambda_{1} \\
& \Re_{1}=b\left(-4 \Lambda_{2}+3 \Gamma_{0}-\Gamma_{1}\right) \\
& \Re_{2}=b\left(-2 \Lambda_{2}+\Gamma_{1}\right) \\
& \Re_{3}=2 b^{2}\left(\Gamma_{0}+\Gamma_{1}\right)-7 b \Gamma_{1} \\
& \mathfrak{R}_{1}^{[1]}=-\mathfrak{R}_{2}-\mathfrak{R}_{3} \\
& \mathfrak{R}_{\perp}^{[2]}=-\mathfrak{R}_{\perp}^{[3]}+\left(\mathfrak{R}_{2} \mathfrak{Y}-\mathfrak{R}_{3} \Gamma_{0}\right) / \Lambda_{2} \\
& \mathfrak{R}_{\perp}^{[3]}=-\mathfrak{R}_{1}(b) / \Gamma_{0}-\mathfrak{R}_{2} \mathfrak{Z}_{1}-\mathfrak{R}_{3}\left(1-\mathfrak{A}_{2}\right) \\
& \mathfrak{R}_{1}^{\bar{N} G}=2 \Lambda_{2}+\Lambda_{3} \\
& \mathfrak{R}_{2}^{N G}=\Lambda_{1} \\
& \mathfrak{T}_{1}=-\Lambda_{3}+\mathfrak{E}_{1}-\mathfrak{E}_{3}+b\left(\mathfrak{B}_{2}-\mathfrak{B}_{1}\right) \\
& \mathfrak{T}_{2}=\Gamma_{0}+\mathfrak{E}_{1}-b \mathfrak{B}_{1} \\
& \mathfrak{Z}_{1}=1+\left(\Gamma_{1}-2\right) / \Gamma_{0} \\
& \mathfrak{Z}_{2}=\mathfrak{Z}_{1}+\Lambda_{3} / \Lambda_{2} \\
& 1 / 2-b / 4 \\
& -b / 2 \\
& -1 / 4-17 / 48 b \\
& -3 / 4+13 / 48 b \\
& -1 / 2 \\
& -3 b / 2 \\
& 1-3 b / 4 \\
& -3 / 2 \\
& -3 / 4 \\
& 1+3 b / 4 \\
& -b / 2 \\
& 1-b / 4 \\
& -b / 2 \\
& -1 / 2 \\
& b / 2 \\
& O\left(b^{2}\right) \\
& -3 b / 4 \\
& O(b) \\
& -3 / 4 \\
& 3 / 4 \\
& -1 / 2 \\
& -3 / 4 \\
& -3 b / 2 \\
& -b \\
& -3 b / 2 \\
& -3 b / 2 \\
& 1 \\
& 3 b \\
& O\left(b^{2}\right) \\
& -3 b^{2} / 2 \\
& O\left(b^{2}\right) \\
& 7 b / 2 \\
& -3 b \\
& 3 b \\
& 1-3 b / 2 \\
& O\left(b^{2}\right) \\
& 1-5 b / 4 \\
& -1-5 b / 2 \\
& -7 b / 4
\end{aligned}
$$$$
\text { behavior for } b \rightarrow 0
$$$$
1-3 b / 2
$$$$
b(1-3 b / 2)
$$$$
b(1-3 b / 4)
$$

behavior for $b \rightarrow 0$

TABLE 1 . Bessel coefficients and their asymptotic behavior for $b \rightarrow 0$.

form

$$
\begin{aligned}
\mathscr{C}_{2}= & \frac{\bar{T}_{\perp p}}{m_{p} \Omega_{p}^{2}}\left(\bar{p}_{\perp p}-\bar{p}_{\| p}\right) \widehat{\mathfrak{C}}_{3} \partial_{\|} \frac{|\boldsymbol{B}|}{B_{0}}+\frac{\bar{p}_{\| p}}{\Omega_{p}} \widehat{\mathfrak{C}_{2}}\left(\frac{c \boldsymbol{E}_{H}}{B_{0}} \cdot \boldsymbol{b}\right) \\
& -\frac{\bar{T}_{\perp p}}{m_{p} \Omega_{p}^{3}}\left(\bar{p}_{\perp p}-2 \bar{p}_{\| p}\right) \widehat{\mathfrak{C}_{4}} \frac{c}{B_{0}} \partial_{\|}(\boldsymbol{n}: \nabla \boldsymbol{E})
\end{aligned}
$$


and $\dagger$

$$
\begin{aligned}
\mathscr{D}_{2}= & -\frac{\bar{p}_{\perp p}}{\Omega_{p}}\left[\widehat{\mathfrak{D}_{3}}-\frac{\bar{\beta}_{\perp}}{2\left(1+\bar{\beta}_{\perp}\right)} \widehat{\mathfrak{D}_{3} \mathfrak{T}_{1}}\right]\left(\boldsymbol{u}_{p} \cdot \boldsymbol{b}\right)+\frac{v_{\text {th } \| p}}{\Omega_{p}} \frac{\mathrm{H}}{\sqrt{\pi}}\left[\bar{p}_{\perp p} \widehat{\mathfrak{D}_{3}}-\frac{\bar{p}_{\| p}}{2} \widehat{\mathfrak{D}_{6} \mathfrak{Z}_{1}}\right] \frac{T_{\perp p}}{\bar{T}_{\perp p}} \\
& +\frac{v_{\text {th } \| p}}{\Omega_{p}} \frac{\mathrm{H}}{\sqrt{\pi}}\left[\bar{p}_{\perp p}\left(\frac{\bar{T}_{\perp p}}{\bar{T}_{\| p}}-1\right) \widehat{\mathfrak{D}_{3} \Gamma_{0}}-\frac{\bar{p}_{\| e}}{2}\left(\frac{\bar{T}_{\perp e}}{\bar{T}_{\| e}}-1\right) \widehat{\mathfrak{D}}_{6}\right] \frac{|\boldsymbol{B}|}{B_{0}} \\
& -\frac{v_{\text {th } \| p}}{\Omega_{p}^{2}} \frac{\mathrm{H}}{\sqrt{\pi}}\left[\bar{p}_{\perp p} \widehat{\mathfrak{D}}_{3}-\frac{\bar{p}_{\| p}}{2} \widehat{\mathfrak{D}_{6} \mathfrak{Z}_{2}}\right]\left(\nabla \wedge \boldsymbol{u}_{p}\right) \cdot \boldsymbol{b}+\frac{\bar{p}_{\perp p}}{\Omega_{p}^{2}}\left[v_{\Delta p}^{2}\left(\widehat{\mathfrak{D}}_{3}+\widehat{\mathfrak{D}_{5}}\right)\right. \\
& \left.-\left(v_{A}^{2}+v_{\Delta e}^{2}\right)\left(\mathfrak{T}_{2} \widehat{-1}\right) \mathfrak{D}_{3}+\left(v_{A}^{2}+\frac{\bar{p}_{\perp e}}{\rho_{0}}+v_{\Delta e}^{2}\right) \frac{\widehat{\mathfrak{D}_{3} \mathfrak{T}_{1}}}{1+\bar{\beta}_{\perp}}\right]\left(\nabla \wedge \frac{\boldsymbol{B}}{B_{0}}\right) \cdot \boldsymbol{b} \\
& -\frac{v_{\text {th } \| p} \bar{p}_{\| p}}{2 \Omega_{p}} \frac{\mathrm{H}}{\sqrt{\pi}} \widehat{\mathfrak{D}_{6}}\left[\frac{p_{\| e}}{\bar{p}_{\| p}}+\frac{\rho_{p}}{\rho_{0}}\right]+\frac{\bar{p}_{\perp p}}{\Omega_{p} \rho_{0} v_{A}^{2}} \frac{\widehat{\mathfrak{D}_{3} \mathfrak{T}_{1}}}{1+\bar{\beta}_{\perp}}\left(q_{\perp p}+q_{\perp e}\right) \\
& +\frac{\bar{p}_{\perp p}}{\Omega_{p}^{3}}\left(v_{\Delta p}^{2}-\frac{\bar{p}_{\| p}}{\rho_{0}}\right) \widehat{\mathfrak{D}_{3}} \partial_{\|}\left[\left(\nabla \wedge \frac{c \boldsymbol{E}}{B_{0}}\right) \cdot \boldsymbol{b}\right],
\end{aligned}
$$

where $v_{\Delta r}^{2}=\left(\bar{p}_{\perp r}-\bar{p}_{\| r}\right) / \rho_{0}$ and $\bar{\beta}_{\perp}=8 \pi\left(\bar{p}_{\perp p}+\bar{p}_{\perp e}\right) / B_{0}^{2}$. It may furthermore be useful in (5.10) and (5.11) to make the same replacements for $\frac{c}{B_{0}} \boldsymbol{n}: \nabla \boldsymbol{E}$ and $\nabla \wedge \frac{c \boldsymbol{E}}{B_{0}} \cdot \boldsymbol{b}$ as described just before (5.7).

As already mentioned, when addressing questions such as plasma heating (Laveder et al. 2013), all the terms involving $\Pi$ (and then also $\boldsymbol{S}_{\perp r}^{\|}$and $\boldsymbol{S}_{\perp r}^{\perp}$ ), are in contrast to be retained in the gyrotropic pressure equations. In this case, we write $\Pi=$ $\Pi_{\perp}+\boldsymbol{b} \otimes \boldsymbol{\Pi}_{\|}+\boldsymbol{\Pi}_{\|} \otimes \boldsymbol{b}$ where the tensor $\Pi_{\perp}$ is given by (D 4), with here $-\Delta_{\perp}^{-1}=\widehat{\frac{1}{k \cdot \bar{n} \cdot \boldsymbol{k}}}$, and the condition that, in Fourier space, $\Pi_{\perp}$ vanishes when $\boldsymbol{k} \cdot \overline{\boldsymbol{n}} \cdot \boldsymbol{k}=0$.

\subsubsection{Non-gyrotropic heat fluxes}

We write

$$
\boldsymbol{S}_{\perp r}^{\perp}=-\left(\boldsymbol{n} \cdot \nabla \mathscr{E}_{r}+\boldsymbol{b} \wedge \nabla \mathscr{F}_{r}\right),
$$

where (again isolating the Hall electric field contribution)

$$
\begin{aligned}
& \mathscr{E}_{p}=-\frac{\bar{p}_{\perp p} \bar{T}_{\perp p}}{m_{p} \Omega_{p}^{2}}\left[\widehat{\mathfrak{E}_{4}}\left(\nabla \wedge \frac{c \boldsymbol{E}_{H}}{B_{0}}\right) \cdot \boldsymbol{b}+\widehat{\mathfrak{E}_{6}} \boldsymbol{n}: \nabla \boldsymbol{u}_{p}\right] \\
& \mathscr{F}_{p}=\frac{2 \bar{p}_{\perp p} \bar{T}_{\perp p}}{m_{p} \Omega_{p}}\left[-\left(\widehat{1+\mathfrak{A}_{2}}\right) \frac{T_{\perp p}^{\prime}}{\bar{T}_{\perp p}}+\frac{1}{2 \Omega_{p}} \widehat{\mathfrak{F}_{1}}\left(\nabla \wedge \boldsymbol{u}_{p}\right) \cdot \boldsymbol{b}\right],
\end{aligned}
$$

together with $\mathscr{E}_{e}=0$ and $\mathscr{F}_{e}=2 \frac{\bar{p}_{\perp e}}{m_{p} \Omega_{p}} T_{\perp e}^{\prime}$. Similarly,

$$
\boldsymbol{S}_{\perp p}^{\|}=-\frac{v_{\mathrm{th} \| p}^{2}}{\Omega_{p}}\left(\bar{p}_{\perp p}-\bar{p}_{\| p}\right) \boldsymbol{b} \wedge \partial_{\|} \boldsymbol{b}-\boldsymbol{n} \cdot \nabla \mathscr{G}_{2 p}-\boldsymbol{b} \wedge \nabla \mathscr{H}_{2 p},
$$

$\dagger$ Note a misprint in (B9) of Passot et al. (2012) that should read $\frac{\mathscr{T}}{i \|_{\ddagger}}=\bar{p}_{\perp p}\left\{\mathfrak{T}_{1}(b) \frac{i k_{\perp} \cdot \boldsymbol{u}_{\perp p}}{i k_{z}}+\right.$ $\left.\left[\mathfrak{T}_{2}(b)\left(1+\frac{v_{\Delta e}^{2}}{v_{A}^{2}}\right)-\left(1+\frac{v_{\Delta p}^{2}}{v_{A}^{2}}+\frac{v_{\Delta e}^{2}}{v_{A}^{2}}\right)\right] \frac{j_{z}}{n_{0} e}\right\}$. Furthermore, the term $\left(1 / i k_{z}\right)\left(e E_{z} / \bar{T}_{\| p}\right)$ arising in (B8) of this reference is determined through linearization of the electric field in the form $\frac{1}{i k_{z}} \frac{e E_{z}}{\bar{T}_{\| p}} \approx$ $-\frac{p_{\| e}^{\prime}}{\bar{p}_{\| p}}+\frac{\left(\bar{p}_{\| e}-\bar{p}_{\perp e}\right)}{\bar{p}_{\| p}} \frac{B_{z}^{\prime}}{B_{0}}$. 
where again the first term originates from a combination of the singular terms in the potentials $\mathscr{G}$ and $\mathscr{H}$ defined in Passot et al. (2012). Note that, in order to ensure a better matching with the large-scale asymptotic theory, we use here an expression of $\mathscr{H}_{2 p}$, derived in Appendix C, which is different from that given in Passot et al. (2012). We have

$$
\begin{aligned}
\mathscr{G}_{2 p}= & \frac{v_{\mathrm{th} \| p}^{2} \bar{T}_{\perp p}}{m_{p} \Omega_{p}^{3}}\left(\bar{p}_{\perp p}-\bar{p}_{\| p}\right) \widehat{\mathfrak{G}_{2}} \partial_{\|}\left[\boldsymbol{b} \cdot\left(\nabla \wedge \frac{\boldsymbol{B}}{B_{0}}\right)\right] \\
\mathscr{H}_{2 p}= & -\frac{\bar{p}_{\perp p}}{m_{p} \Omega_{p}} T_{\| p}^{\prime}-\frac{\widetilde{r}_{\| \perp p}}{\Omega_{p}}+\frac{\bar{p}_{\perp p}}{\Omega_{p}} \frac{v_{\mathrm{th} \| \mathrm{p}}^{2}}{2}\left\{\widehat{\mathfrak{H}_{1}} \frac{T_{\| p}}{\bar{T}_{\| p}}+\widehat{\mathfrak{H}_{2}}\left[\left(\frac{p_{\| e}}{\bar{p}_{\| p}}-\frac{\left(\bar{p}_{\| e}-\bar{p}_{\perp e}\right)}{\bar{p}_{\| p}} \frac{|\boldsymbol{B}|}{B_{0}}\right)\right.\right. \\
& \left.\left.+\frac{\rho}{\rho_{0}}+\widehat{\mathfrak{H}_{3}} \frac{T_{\perp p}}{\bar{T}_{\perp p}}-\frac{1}{\Omega_{p}} \widehat{\mathfrak{H}_{4}}\left(\nabla \wedge \boldsymbol{u}_{p}\right) \cdot \boldsymbol{b}\right]\right\}
\end{aligned}
$$

For the electrons, the formula simplifies into

$$
\boldsymbol{S}_{\perp e}^{\|}=\frac{v_{\mathrm{th} \| p}^{2}}{\Omega_{p}} \frac{\bar{T}_{\| e}}{\bar{T}_{\| p}}\left(\bar{p}_{\perp e}-\bar{p}_{\| e}\right) \boldsymbol{b} \wedge \partial_{\|} \boldsymbol{b}-\frac{v_{\mathrm{th} \| p}^{2}}{2 \Omega_{p}} \bar{p}_{\perp e} \boldsymbol{b} \wedge \nabla\left(\frac{T_{\| e}}{\bar{T}_{\| p}}\right) .
$$

Note that, as the present description of the kinetic effects is only linearly accurate, one can consistently neglect the contributions of terms of the form $\nabla \cdot(\boldsymbol{b} \wedge \nabla f)$ arising in $\nabla \cdot S_{\perp r}^{\|}$and $\nabla \cdot S_{\perp r}^{\perp}$.

\subsubsection{Non-gyrotropic 4th-rank cumulants}

For the non-gyrotropic contributions to the fourth-rank cumulants, we have $R_{\| r}^{N G}=$ 0 , together with†

$$
\begin{aligned}
& \frac{p_{\perp p}}{\rho_{p}} \nabla \cdot(\boldsymbol{n} \cdot \Pi)+R_{\perp p}^{N G} \approx \frac{\bar{T}_{\perp p}}{m_{p}}\left(\nabla_{\perp} \cdot \Pi_{\perp}\right)+R_{\perp p}^{N G} \\
& \left.=-\frac{\bar{T}_{\perp p}}{m_{p}}\left(\bar{p}_{\perp p}-\bar{p}_{\| p}\right) \widehat{\widehat{\mathfrak{R}_{1}^{N G}} \partial_{\|}} \frac{|\boldsymbol{B}|}{B_{0}}-\frac{1}{\Omega_{p}} \mathfrak{R}_{2}^{N G} k_{\perp}^{2} \frac{c \boldsymbol{E}_{H} \cdot \boldsymbol{b}}{B_{0}}\right] \\
& \quad-\frac{\bar{T}_{\perp p}}{m_{p} \Omega_{p}}\left(\bar{p}_{\perp p}-2 \bar{p}_{\| p}\right) \widehat{\mathfrak{R}_{2}^{N G}} \boldsymbol{b} \cdot\left[\nabla\left(\nabla \cdot \frac{c \boldsymbol{E}}{B_{0}}\right)-\Delta \frac{c \boldsymbol{E}}{B_{0}}\right]
\end{aligned}
$$

and

$$
\begin{aligned}
R_{\perp e}^{N G}= & \frac{\bar{T}_{\perp e}}{m_{p} \Omega_{p}}\left(\bar{p}_{\perp e}-\bar{p}_{\| e}\right)\left(\widehat{-k_{\perp}^{2}}\right)\left[\frac{3 \bar{T}_{\perp e}}{m_{p} \Omega_{p}} \partial_{\|} \frac{|\boldsymbol{B}|}{B_{0}}+\frac{c\left(\boldsymbol{E}_{H} \cdot \boldsymbol{b}\right)}{B_{0}}\right] \\
& +\frac{\bar{T}_{\perp e}}{m_{p} \Omega_{p}}\left(\bar{p}_{\perp e}-2 \bar{p}_{\| e}\right) \boldsymbol{b} \cdot\left[\nabla\left(\nabla \cdot \frac{c \boldsymbol{E}}{B_{0}}\right)-\Delta \frac{c \boldsymbol{E}}{B_{0}}\right] .
\end{aligned}
$$

As previously, it may be convenient to rewrite the last terms of the two above equations by isolating the contribution of the Hall electric field and simplifing the resulting expressions in a way consistent with the linear kinetic theory. This leads to replace $\boldsymbol{b} \cdot\left[\nabla\left(\nabla \cdot \frac{c \boldsymbol{E}}{B_{0}}\right)-\Delta \frac{c \boldsymbol{E}}{B_{0}}\right]$ by $-\partial_{\|}\left[\boldsymbol{b} \cdot\left(\nabla \wedge \boldsymbol{u}_{p}\right)\right]+\partial_{\|}\left(\nabla \cdot \frac{c \boldsymbol{E}_{H}}{B_{0}}\right)-\Delta\left(\boldsymbol{b} \cdot \frac{c \boldsymbol{E}_{H}}{B_{0}}\right)$, which appears preferable at the level of numerical coding.

$\dagger$ The present definition of $\Re_{2}^{N G}(b)$ differs from that given Passot and Sulem (2007) and Passot et al. (2012) where an additional factor $b$ is included. 


\subsubsection{A simplified closure for the electrons}

It turns out that the simplified model where, instead of obeying dynamical equations, the electron gyrotropic fluxes are given by equations (3.17) and (3.19), leads to results that are essentially identical to those obtained by closing at the level of the fourthrank moments for both particle species. An advantage of this description is that the terms involving the ion-electron mass ratio become purely dissipative. In numerical simulations, the linear contribution of these terms can then be easily handled implicitly (thus permitting a significant increase of the time step), after replacing, for the electrons, pressure by temperature equations.

\subsection{Matching large-scale asymptotics and sub-ion-scale kinetic closure}

In Sec. 3, we presented expressions of the non-gyrotropic moments (hereafter indicated by the superscripts LS), derived in a large-scale fully-nonlinear asymptotics, while the corresponding formulas given in Sec. 5.1 are closure relations based on the linear low-frequency kinetic theory. One easily checks that the two descriptions overlap, when linearized about a homogeneous equilibrium plasma with a uniform ambient field and taken to leading-order in a large-scale expansion. This suggests to isolate, in the kinetic-based closures, the leading order large-scale contributions to the nongyrotropic pressure and heat fluxes, and replace them by the fully nonlinear large-scale expressions given in Sec. 3. This results in formulas which are asymptotically exact to leading order in a large-scale asymptotics, but also reproduce at all the scales the linear properties of the low-frequency modes. Such a model provides in particular a relatively accurate description of the work of the non-gyrotropic pressure force. At the linear level, it identifies with the model discussed in Sec. 5.1, which was extensively tested in Passot et al. (2012) and Hunana et al. (2013a,b). The closure of the hierarchy equations for the gyrotropic moments is indeed done as in Sec. 5.1, either at the level of the fourth order cumulants for both the ions and the electrons or, more conveniently, by closing the electron hierarchy at the level of the heat fluxes, as previously discussed. The non-gyrotropic moments then take the form described below.

\subsubsection{Ion non-gyrotropic pressure tensor}

We write

$$
\nabla \cdot \Pi=\nabla \cdot \Pi^{L S}+\nabla \cdot\left[-\mathscr{A}^{S S} \boldsymbol{n}+\mathscr{B}^{S S} \epsilon \cdot \boldsymbol{b}+\boldsymbol{b} \otimes \boldsymbol{\Pi}_{\|}^{S S}+\boldsymbol{\Pi}_{\|}^{S S} \otimes \boldsymbol{b}\right] .
$$

Here $\Pi^{L S}$ is given by (3.1)-(3.5) where, in spite of the fluid hierarchy closure performed at the level of the fourth-rank moments, the perpendicular heat flux $q_{\perp p}$ is given by equation (3.18), in order to ensure a correct matching with the small-scale formula. Furthermore,

$$
\begin{aligned}
& \mathscr{A}^{S S}=\bar{p}_{\perp p}\left[\frac{1}{\Omega_{p}}\left(\widehat{\mathfrak{A}_{1}-\frac{1}{2}}\right) \boldsymbol{b} \cdot\left(\nabla \wedge \boldsymbol{u}_{p}\right)+\widehat{\mathfrak{A}_{2}} \frac{T_{\perp p}}{\bar{T}_{\perp p}}\right] \\
& \mathscr{B}^{S S}=\frac{\bar{p}_{\perp p}}{\Omega_{p}}\left[-\widehat{\mathfrak{B}}_{1} \boldsymbol{b} \cdot\left(\nabla \wedge \frac{c \boldsymbol{E}_{H}}{B_{0}}\right)+\left(\widehat{\mathfrak{B}_{3}+\frac{1}{2}}\right) \boldsymbol{n}: \nabla \boldsymbol{u}_{p}\right],
\end{aligned}
$$

and

$$
\boldsymbol{\Pi}_{\|}^{S S}=-\boldsymbol{n} \cdot \nabla \mathscr{C}_{2}-\boldsymbol{b} \wedge \nabla \mathscr{D}_{2}^{S S}
$$


where

$$
\begin{aligned}
\mathscr{D}_{2}^{S S}= & -\frac{\bar{p}_{\perp p}}{\Omega_{p}}\left[\left(\widehat{\mathfrak{D}_{3}-} 1\right)-\frac{\bar{\beta}_{\perp}}{2\left(1+\bar{\beta}_{\perp}\right)} \widehat{\mathfrak{D}_{3} \mathfrak{T}_{1}}\right]\left(\boldsymbol{u}_{p} \cdot \boldsymbol{b}\right) \\
& +\frac{v_{\mathrm{th} \| p}}{\Omega_{p}} \frac{\mathrm{H}}{\sqrt{\pi}}\left[\bar{p}_{\perp p}\left(\widehat{\mathfrak{D}_{3}-} 1\right)-\frac{\bar{p}_{\| p}}{2} \widehat{\mathfrak{D}_{6} \mathfrak{Z}_{1}}\right] \frac{T_{\perp p}}{\bar{T}_{\perp p}} \\
& +\frac{v_{\text {th } \| p}}{\Omega_{p}} \frac{\mathrm{H}}{\sqrt{\pi}}\left[\bar{p}_{\perp p}\left(\frac{\bar{T}_{\perp p}}{\bar{T}_{\| p}}-1\right)\left(\mathfrak{D}_{3} \widehat{\Gamma_{0}}-1\right)-\frac{\bar{p}_{\| e}}{2}\left(\frac{\bar{T}_{\perp e}}{\bar{T}_{\| e}}-1\right) \widehat{\mathfrak{D}_{6}}\right] \frac{|\boldsymbol{B}|}{B_{0}} \\
& -\frac{v_{\mathrm{th} \| p}}{\Omega_{p}^{2}} \frac{\mathrm{H}}{\sqrt{\pi}}\left[\bar{p}_{\perp p}\left(\widehat{\mathfrak{D}_{3}-} 1\right)-\frac{\bar{p}_{\| p}}{2} \widehat{\mathfrak{D}_{6} \mathfrak{Z}_{2}}\right]\left(\nabla \wedge \boldsymbol{u}_{p}\right) \cdot \boldsymbol{b} \\
& +\frac{\bar{p}_{\perp p}}{\Omega_{p}^{2}}\left[v_{\Delta p}^{2}\left(\mathfrak{D}_{3}-\widehat{1}+\mathfrak{D}_{5}\right)-\left(v_{A}^{2}+v_{\Delta e}^{2}\right)\left(\mathfrak{T}_{2}-\widehat{-1}\right) \mathfrak{D}_{3}\right. \\
& \left.+\left(v_{A}^{2}+\frac{\bar{p}_{\perp e}}{\rho_{0}}+v_{\Delta e}^{2}\right) \frac{\mathfrak{D}_{3} \mathfrak{T}_{1}}{1+\bar{\beta}_{\perp}}\right]\left(\nabla \wedge \frac{\boldsymbol{B}}{B_{0}}\right) \cdot \boldsymbol{b}-\frac{v_{\mathrm{th} \| p} \bar{p}_{\| p}}{2 \Omega_{p}} \frac{\mathrm{H}}{\sqrt{\pi}} \widehat{\mathfrak{D}_{6}}\left[\frac{p_{\| e}}{\bar{p}_{\| p}}+\frac{\rho_{p}}{\rho_{0}}\right] \\
& +\frac{\bar{p}_{\perp p}}{\Omega_{p} \rho_{0} v_{A}^{2}} \frac{\widehat{D}_{3} \mathfrak{T}_{1}}{1+\bar{\beta}_{\perp}}\left(q_{\perp p}+q_{\perp e}\right)+\frac{\bar{p}_{\perp p}}{\Omega_{p}^{3}}\left(v_{\Delta p}^{2}-\frac{\bar{p}_{\| p}}{\rho_{0}}\right) \widehat{\mathfrak{D}_{3}} \partial_{\|}\left[\left(\nabla \wedge \frac{c \boldsymbol{E}}{B_{0}}\right) \cdot \boldsymbol{b}\right] .
\end{aligned}
$$

Again in the last term $\left(\nabla \wedge \frac{c \boldsymbol{E}}{B_{0}}\right) \cdot \boldsymbol{b}$ can be replaced as indicated before (5.7).

Similarly, the full gyroviscous tensor is rewritten $\Pi=\Pi^{L S}+\Pi^{S S}$, where

$$
\Pi^{S S}=\Pi_{\perp}^{S S}+\boldsymbol{b} \otimes \boldsymbol{\Pi}_{\|}^{S S}+\boldsymbol{\Pi}_{\|}^{S S} \otimes \boldsymbol{b} .
$$

Defining the tensor $\boldsymbol{r}=\boldsymbol{b} \cdot \epsilon$, we have

$$
\Pi_{\perp i j}^{S S}=\left[n_{i p} n_{j q}-r_{i p} r_{j q}\right] \partial_{p} \partial_{q}\left(-\Delta^{-1} \mathscr{A}^{S S}\right)+\left(n_{i p} r_{j q}+n_{j p} r_{i q}\right) \partial_{p} \partial_{q}\left(-\Delta^{-1} \mathscr{B}^{S S}\right),
$$

with

$$
\begin{aligned}
-\Delta^{-1} \mathscr{A}^{S S} & =\frac{\bar{p}_{\perp p} \bar{T}_{\perp p}}{m_{p} \Omega_{p}^{2}}\left[\frac{1}{\Omega_{p}}\left[\frac{1}{b}\left(\widehat{\mathfrak{A}_{1}}-\frac{1}{2}\right)\right] \boldsymbol{b} \cdot\left(\nabla \wedge \boldsymbol{u}_{p}\right)+\widehat{\left[\frac{\mathfrak{A}_{2}}{b}\right]} \frac{T_{\perp p}^{\prime}}{\bar{T}_{\perp p}}\right] \\
-\Delta^{-1} \mathscr{B} & =\frac{\bar{p}_{\perp p} \bar{T}_{\perp p}}{m_{p} \Omega_{p}^{3}}\left[-\left[\frac{1}{b} \mathfrak{B}_{1}\right] \boldsymbol{b} \cdot\left(\nabla \wedge \frac{c \boldsymbol{E}_{H}}{B_{0}}\right)+\left[\frac{1}{b}\left(\widehat{\mathfrak{B}_{3}}+\frac{1}{2}\right)\right] \boldsymbol{n}: \nabla \boldsymbol{u}_{p}\right] .
\end{aligned}
$$

\subsubsection{Non-gyrotropic heat fluxes}

As previously we write $S_{\perp p}^{\perp}=S_{\perp p}^{\perp L S}+S_{\perp p}^{\perp S S}$ and $S_{\perp p}^{\|}=S_{\perp p}^{\| L S}+S_{\perp p}^{\| S S}$, where $S_{\perp p}^{\perp L S}$ and $S_{\perp p}^{\| L S}$ are given by equations (3.6) and (3.7), and

$$
\begin{aligned}
& \boldsymbol{S}_{\perp p}^{\perp S S}=-\boldsymbol{n} \cdot \nabla \mathscr{E}_{2 p}-\boldsymbol{b} \wedge \nabla \mathscr{F}_{2}^{S S} \\
& \boldsymbol{S}_{\perp p}^{\| S S}=-\boldsymbol{n} \cdot \nabla \mathscr{G}_{2 p}-\boldsymbol{b} \wedge \nabla \mathscr{H}_{2}^{S S},
\end{aligned}
$$

with

$$
\underset{p}{\mathscr{F}_{p} S S}=\frac{2 \bar{T}_{\perp p} \bar{p}_{\perp p}}{m_{p} \Omega_{p}}\left[-\widehat{\mathfrak{A}}_{2} \frac{T_{\perp p}}{\bar{T}_{\perp p}}+\frac{1}{2 \Omega_{p}} \widehat{\mathfrak{F}}_{1}\left(\nabla \wedge \boldsymbol{u}_{p}\right) \cdot \boldsymbol{b}\right]
$$




$$
\begin{aligned}
\mathscr{H}_{2 p}= & \frac{\bar{p}_{\perp p}}{\Omega_{p}} \frac{v_{\mathrm{th} \| \mathrm{p}}^{2}}{2}\left\{\widehat{\mathfrak{H}}_{1} \frac{T_{\| p}}{\bar{T}_{\| p}}+\widehat{\mathfrak{H}_{2}}\left[\left(\frac{p_{\| e}}{\bar{p}_{\| p}}-\frac{\left(\bar{p}_{\| e}-\bar{p}_{\perp e}\right)}{\bar{p}_{\| p}} \frac{|\boldsymbol{B}|}{B_{0}}\right)\right.\right. \\
& \left.\left.+\frac{\rho}{\rho_{0}}+\widehat{\mathfrak{H}_{3}} \frac{T_{\perp p}}{\bar{T}_{\perp p}}-\frac{1}{\Omega_{p}} \widehat{\mathfrak{H}_{4}}\left(\nabla \wedge \boldsymbol{u}_{p}\right) \cdot \boldsymbol{b}\right]\right\} .
\end{aligned}
$$

In this linear description of the small scales, the contributions of $\mathscr{F}_{2 p}^{S S}$ and $\mathscr{H}_{2 p}^{S S}$ can be neglected in evaluating $\nabla \cdot S_{\perp p}^{\| S S}$ and $\nabla \cdot S_{\perp p}^{\perp S S}$. Furthermore, in the present model, the contribution of $\sigma_{p}$ to the ion heat flux tensor identifies with its large-scale expression. The electron non-gyrotropic heat flux vectors $S_{\perp e}^{\|}$and $S_{\perp e}^{\perp}$ also reduce to the large-scale contributions.

\section{Conclusion}

We have presented kinetic-based closures of the fluid hierarchy, together with various possible descriptions of the ion FLR corrections. A model including a fully nonlinear description of the non-gyrotropic moments, computed to leading order in a large-scale asymptotics, and involving a closure of the fluid hierarchy at the level of the ion and electron heat fluxes, was in particular found to provide unexpectedly good results. It indeed leads to an accurate description of collisionless plasmas with weak temperature anisotropy, when concentrating on the dispersion and damping of kinetic Alfvén waves, even at scales that may be smaller than the ion gyroscale, thus significantly beyond the supposed validity range of the asymptotics. A main observation concerning this approach is the need of consistently including the first order corrections for all the retained moments, in order to prevent spurious instabilities in the presence of Landau damping. Large-scale models nevertheless fail to provide a satisfactory description of the mirror instability that develops in the presence of a temperature anisotropy. This micro-instability is in contrast well reproduced by the FLR-Landau fluid model (Passot and Sulem 2007; Passot et al. 2012), which involves a linear description of the FLR corrections based on the low-frequency kinetic theory. This approach is here revisited in order to take into account the local distortion of the magnetic field lines in the expressions of the kinetic quantities, a procedure needed to ensure well-posedness in the nonlinear regime. Such a model was used in Passot et al. (2014) to perform the first fluid simulations of the ion dissipation range. We also propose a new approach where a linearly accurate modeling of the small-scale FLR corrections is supplemented to their fully nonlinear description at large scales. It is also of interest to point out that both with the previously developed FLR-Landau fluid model and the present matching procedure, a satisfactory accuracy is obtained when the fluid hierarchy for the ions is closed at the level of the fourth-rank moments, and that for the electrons at the level of the heat fluxes. Numerically, this description has the main advantage of permitting an easy implementation of a semi-implicit scheme for the contributions associated with electron Landau damping, which significantly reduces the constraint on the time step prescribed in a purely explicit scheme by the large value of the proton-electron mass ratio.

Finally, we note that, except for the Landau damping term, the large-scale model is local in physical space, which is a convenient feature in the perspective of finitedifference numerical simulations on computers with a massively parallel architecture. In this context, the possibility of designing a satisfactory local approximation of the Landau operator appears an interesting challenge, in particular when planning to retain nontrivial boundary conditions. Differently, the FLR-Landau fluid and the 
model based on the matching procedure involve non-local operators in Fourier space and require the use of a pseudo-spectral method in a periodic domain.

\section{Acknowledgements}

We thank Dimitri Laveder for careful reading of the paper and useful comments. The research leading to these results has received funding from the European Commission's Seventh Framework Programme (FP7/2007-2013) under the grant agreement SHOCK (project number 284515).

\section{Appendix A. Large-scale closure for the gyrotropic heat fluxes}

In order to estimate $q_{\| r}$, we start from the kinetic expression given by (B12) of Passot and Sulem (2007), taken in the large-scale limit. Combining with the kinetic expression (B7) of the same reference for the parallel temperature fluctuations $T_{\| r}^{\prime}$ given in the same reference, we are led to

$$
q_{\| r}=\bar{p}_{\| r} v_{\mathrm{th} \| r} \frac{\zeta_{r}\left[1-3 R\left(\zeta_{r}\right)+2 \zeta_{r}^{2} R\left(\zeta_{r}\right)\right]}{1-R\left(\zeta_{r}\right)+2 \zeta_{r}^{2} R\left(\zeta_{r}\right)} \frac{T_{\| r}^{\prime}}{\bar{T}_{\| r}}
$$

Replacing the plasma response function $R\left(\zeta_{r}\right)$ [where $\zeta_{r}=\omega /\left(\left|k_{\|}\right| v_{\text {th } \| r}\right)$ ] by the threepole Padé approximant

$$
R\left(\zeta_{r}\right) \approx \frac{-\zeta_{r} / 2-a_{0}}{\zeta_{r}^{3}-a_{2} \zeta_{r}^{2}-a_{1} \zeta_{r}-a_{0}}
$$

where $a_{0}=i \operatorname{sgn}\left(k_{z}\right) / \sqrt{\pi}, a_{1}=3 / 2$ and $a_{2}=-2 i \operatorname{sgn}\left(k_{z}\right) / \sqrt{\pi}$, we get

$$
q_{\| r}=-\bar{p}_{\perp r} v_{\text {th } \| r} \frac{2 i}{\sqrt{\pi}} \operatorname{sgn}\left(k_{z}\right) \frac{T_{\| r}^{\prime}}{\bar{T}_{\| r}} .
$$

Similarly, we start from the kinetic expression for $q_{\perp r}$ given by (B13) of Passot and Sulem (2007) again taken in the large-scale limit. Here we resorted to replace the plasma response function by a one-pole Padé approximant, thus writing $\zeta_{r} R\left(\zeta_{r}\right) \approx$ $\frac{i}{\sqrt{\pi}} \operatorname{sgn}\left(k_{z}\right)\left(1-R\left(\zeta_{r}\right)\right)$. Using also (24) and (A5) of Passot and Sulem (2007), we finally get

$$
\begin{aligned}
q_{\perp p}= & -\frac{\bar{p}_{\perp p}}{\Omega_{p}} \frac{\left(\bar{p}_{\perp p}-\bar{p}_{\| p}\right)}{\rho_{0}}\left(\nabla_{\perp} \wedge \frac{\boldsymbol{B}_{\perp}}{B_{0}}\right)_{z}-\bar{p}_{\perp p} v_{\text {th } \| \mathrm{p}} \frac{i \operatorname{sgn}\left(k_{z}\right)}{\sqrt{\pi}} \\
& \times\left[\frac{T_{\perp p}}{\bar{T}_{\perp p}}-\frac{1}{\Omega_{p}}\left(\nabla_{\perp} \wedge \boldsymbol{u}_{\perp p}\right)_{z}+\left(\frac{\bar{T}_{\perp p}}{\bar{T}_{\| p}}-1\right) \frac{B_{z}}{B_{0}}\right]
\end{aligned}
$$

and, at scales large relatively to the electron gyroscale,

$$
q_{\perp e}=\frac{\bar{p}_{\perp e}}{\Omega_{p}} \frac{\left(\bar{T}_{\perp e}-\bar{T}_{\| e}\right)}{m_{p}}\left(\nabla \wedge \frac{\boldsymbol{B}_{\perp}}{B_{0}}\right)_{z}-\bar{p}_{\perp e} v_{\mathrm{th} \| \mathrm{e}} \frac{i \operatorname{sgn}\left(k_{z}\right)}{\sqrt{\pi}}\left[\frac{T_{\perp e}}{\bar{T}_{\perp e}}+\left(\frac{\bar{T}_{\perp e}}{\bar{T}_{\| e}}-1\right) \frac{|\boldsymbol{B}|}{B_{0}}\right] .
$$

\section{Appendix B. The 'Bessel coefficients'}

The kinetic-based closures described in Sec. 5 involve various functions, we refer to as Bessel coefficients, which are constructed from the functions $\Gamma_{0}(b)$ and $\Gamma_{1}(b)$. 
They are listed in Table 1, together with their asymptotic behavior in the largescale limit $b \rightarrow 0$, based on the expansion $\Gamma_{0}(b)=1-b+\frac{3}{4} b^{2}-\frac{5}{12} b^{3}+O\left(b^{4}\right)$ and $\Gamma_{1}(b)=\frac{b}{2}-\frac{b^{2}}{2}+\frac{5}{16} b^{3}+O\left(b^{4}\right)$.

\section{Appendix C. An alternative modeling of the $\mathscr{H}_{p}$ potential}

We present here an alternative to the computation of the potential $\mathscr{H}_{p}$ given in Passot et al. (2012), more appropriate for matching with the large-scale asymptotics. For this purpose, we start from the expression of $\mathscr{H}_{p}$ obtained from the lowfrequency linear kinetic theory given by (B11) of Passot and Sulem (2007), that we rewrite $\mathscr{H}_{p}=\mathscr{H}_{1 p}+\mathscr{H}_{2 p}$ with (in Fourier space)

$$
\mathscr{H}_{1 p}=-\frac{2}{\Omega_{p}} \frac{\bar{T}_{\perp p}-\bar{T}_{\| p}}{m_{p}} \frac{k_{z}^{2}}{k_{\perp}^{2}} \frac{B_{z}}{B_{0}}
$$

and

$$
\mathscr{H}_{2 p}=\frac{\bar{p}_{\| p}}{\Omega_{p}} \frac{\bar{T}_{\perp p}}{m_{p}} \frac{\bar{T}_{\perp p}}{\bar{T}_{\| p}}\left(\Gamma_{0}-\Gamma_{1}\right)\left(1-R\left(\zeta_{p}\right)+2 \zeta_{p}^{2} R\left(\zeta_{p}\right)\right)\left(\frac{e \Psi}{\bar{T}_{\perp p}}+2 \frac{B_{z}}{B_{0}}\right),
$$

where $\Psi$ denotes the electric potential defined by $E_{z}=-\partial_{z} \Psi$. Using the kinetic formula for the parallel temperature fluctuations given by (B7) of Passot and Sulem (2007), we write

$$
\mathscr{H}_{2 p}=\frac{\bar{p}_{\| p}}{\Omega_{p}} \frac{\bar{T}_{\perp p}}{m_{p}}\left\{-\frac{T_{\| p}^{\prime}}{\bar{T}_{\| p}}+\frac{\bar{T}_{\perp p}}{\bar{T}_{\| p}}\left(1-R+2 \zeta_{p}^{2} R\right)\left[\left(\Gamma_{0}-\Gamma_{1}\right) \frac{B_{z}}{B_{0}}-\Gamma_{1} \frac{e \Psi}{\bar{T}_{\perp p}}\right]\right\}
$$

or, with the kinetic formula of $\widetilde{r}_{\| \perp p}$ given by (B18) of Passot and Sulem (2007),

$$
\begin{aligned}
\mathscr{H}_{2 p}= & -\frac{\bar{p}_{\perp p}}{m_{p} \Omega_{p}} T_{\| p}^{\prime}-\frac{\widetilde{r}_{\| \perp p}}{\Omega_{p}}+\frac{\bar{p}_{\perp p}}{\Omega_{p}} \frac{\bar{T}_{\perp p}}{m_{p}}\left(1-R+2 \zeta_{p}^{2} R\right) \\
& \times\left\{\left[2 b\left(\Gamma_{0}-\Gamma_{1}\right)-\Gamma_{1}\right] \frac{B_{z}}{B_{0}}+\left[b\left(\Gamma_{0}-\Gamma_{1}\right)-\Gamma_{1}\right] \frac{\bar{T}_{\| p}}{\bar{T}_{\perp p}} \frac{e \Psi}{\bar{T}_{\| p}}\right\} .
\end{aligned}
$$

Using again (B7) of Passot and Sulem (2007) to express

$$
\left(1-R+2 \zeta_{p}^{2} R\right) \frac{B_{z}}{B_{0}}=\frac{\bar{T}_{\| p}}{\bar{T}_{\perp p}} \frac{1}{\Gamma_{0}-\Gamma_{1}}\left(\frac{T_{\| p}^{\prime}}{\bar{T}_{\| p}}+\Gamma_{0}\left(1-R+2 \zeta_{p}^{2} R\right) \frac{e \Psi}{\bar{T}_{\| p}}\right),
$$

we get

$$
\begin{aligned}
\mathscr{H}_{2 p}= & -\frac{\bar{p}_{\perp p}}{m_{p} \Omega_{p}} T_{\| p}^{\prime}-\frac{\widetilde{r}_{\| \perp p}}{\Omega_{p}}+\frac{\bar{p}_{\perp p}}{\Omega_{p}} \frac{v_{\mathrm{th} \| \mathrm{p}}^{2}}{2}\left\{\frac{2 b\left(\Gamma_{0}-\Gamma_{1}\right)-\Gamma_{1}}{\Gamma_{1}-\Gamma_{0}} \frac{T_{\| p}^{\prime}}{\bar{T}_{\| p}}\right. \\
& \left.+\left[\Gamma_{0} \frac{2 b\left(\Gamma_{0}-\Gamma_{1}\right)-\Gamma_{1}}{\Gamma_{1}-\Gamma_{0}}+b\left(\Gamma_{0}-\Gamma_{1}\right)-\Gamma_{1}\right]\left(1+R+2 \zeta_{p}^{2} R\right) \frac{e \Psi}{\bar{T}_{\| p}}\right\},
\end{aligned}
$$

where it is easily checked that $\left(\Gamma_{1}-\Gamma_{0}\right)$ does not vanish.

At this stage, defining $\mathscr{Z}_{1}=\zeta_{p} R \frac{e \Psi}{\bar{T}_{\| p}}$, we are resorted to rewrite

$$
\left(1-R+2 \zeta_{p}^{2} R\right) \frac{e \Psi}{\bar{T}_{\| p}}=\frac{1-R+2 \zeta_{p}^{2} R}{\zeta_{p} R} \mathscr{Z}_{1}
$$


where, using a one-pole Padé approximant for $R$, the first factor in the right-hand side is replaced by $-\sqrt{\pi} \mathrm{H}$ and the second one is given by (8) of Passot et al. (2012) in the form

$$
\begin{aligned}
\mathscr{Z}_{1}= & \frac{\mathrm{H}}{\sqrt{\pi}}\left\{-\frac{1}{i k_{z}} \frac{e E_{z}}{\bar{T}_{\| p}}+\frac{\rho_{p}^{\prime}}{\rho_{0}}+\left(\frac{\Gamma_{1}-2}{\Gamma_{0}}+1\right) \frac{T_{\perp p}^{\prime}}{\bar{T}_{\perp p}}\right. \\
& \left.-\frac{1}{\Omega_{p}}\left(\frac{1-\Gamma_{0}}{b\left(\Gamma_{0}-\Gamma_{1}\right)}+\frac{\Gamma_{1}-2}{\Gamma_{0}}+1\right)\left(i \vec{k}_{\perp} \times \vec{u}_{\perp p}\right)_{z}\right\},
\end{aligned}
$$

with

$$
\frac{1}{i k_{z}} \frac{e E_{z}}{\bar{T}_{\| p}} \approx-\left(\frac{p_{\| e}^{\prime}}{\bar{p}_{\| p}}-\frac{\left(\bar{p}_{\| e}-\bar{p}_{\perp e}\right)}{\bar{p}_{\| p}} \frac{B_{z}^{\prime}}{B_{0}}\right) .
$$

We then easily get (5.17).

\section{Appendix D. Intrinsic form of the non-gyrotropic pressure tensor}

Let us consider the tensor $\Pi_{\perp}$ that in the linear description is characterized in Fourier space by the elements (Passot et al. 2012)

$$
\begin{aligned}
& \Pi_{x x}=-\Pi_{y y}=-\frac{k_{x}^{2}-k_{y}^{2}}{k_{\perp}^{2}} \mathscr{A}+2 \frac{k_{x} k_{y}}{k_{\perp}^{2}} \mathscr{B} \\
& \Pi_{y x}=\Pi_{x y}=-2 \frac{k_{x} k_{y}}{k_{\perp}^{2}} \mathscr{A}-\frac{k_{x}^{2}-k_{y}^{2}}{k_{\perp}^{2}} \mathscr{B},
\end{aligned}
$$

with respect to an orthonormal basis $\left(\boldsymbol{e}_{1}, \boldsymbol{e}_{2}, \boldsymbol{e}_{3}\right)$ where $\boldsymbol{e}_{3}$ is taken along the ambient magnetic field. We define $\widehat{\boldsymbol{k}}_{\perp}=\boldsymbol{k}_{\perp} /\left|k_{\perp}\right|=\cos \psi \boldsymbol{e}_{1}+\sin \psi \boldsymbol{e}_{2}$ and $\widehat{\boldsymbol{k}}_{\perp}^{\perp}=\widehat{\boldsymbol{k}}_{\perp} \wedge \boldsymbol{e}_{3}=$ $\sin \psi \boldsymbol{e}_{1}-\cos \psi \boldsymbol{e}_{2}$. The transverse basis vectors then read $\boldsymbol{e}_{1}=\cos \psi \widehat{\boldsymbol{k}}_{\perp}+\sin \psi \widehat{\boldsymbol{k}}_{\perp}^{\perp}$ and $\boldsymbol{e}_{2}=\sin \psi \widehat{\boldsymbol{k}}_{\perp}-\cos \psi \widehat{\boldsymbol{k}}_{\perp}^{\perp}$. This leads to

$$
\begin{aligned}
\Pi_{\perp}= & (-\cos 2 \psi \mathscr{A}+\sin 2 \psi \mathscr{B})\left(\boldsymbol{e}_{1} \otimes \boldsymbol{e}_{1}-\boldsymbol{e}_{2} \otimes \boldsymbol{e}_{2}\right) \\
& -(\sin 2 \psi \mathscr{A}+\cos 2 \psi \mathscr{B})\left(\boldsymbol{e}_{1} \otimes \boldsymbol{e}_{2}+\boldsymbol{e}_{2} \otimes \boldsymbol{e}_{1}\right) \\
= & -\mathscr{A}\left(\widehat{\boldsymbol{k}}_{\perp} \otimes \widehat{\boldsymbol{k}}_{\perp}-\widehat{\boldsymbol{k}}_{\perp}^{\perp} \otimes \widehat{\boldsymbol{k}}_{\perp}^{\perp}\right)+\mathscr{B}\left(\widehat{\boldsymbol{k}}_{\perp} \otimes \widehat{\boldsymbol{k}}_{\perp}^{\perp}+\widehat{\boldsymbol{k}}_{\perp}^{\perp} \otimes \widehat{\boldsymbol{k}}_{\perp}\right) .
\end{aligned}
$$

In physical space,

$$
\Pi_{\perp}=-\left[\nabla_{\perp} \otimes \nabla_{\perp}-(\boldsymbol{b} \wedge \nabla) \otimes(\boldsymbol{b} \wedge \nabla)\right] \Delta_{\perp}^{-1} \mathscr{A}+\left[\nabla_{\perp} \otimes(\boldsymbol{b} \wedge \nabla)+(\boldsymbol{b} \wedge \nabla) \otimes \nabla_{\perp}\right] \Delta_{\perp}^{-1} \mathscr{B},
$$

whose components are given by (defining the tensor $\boldsymbol{r}=\boldsymbol{b} \cdot \epsilon$ )

$$
\Pi_{\perp i, j}=\left[n_{i p} n_{j q}-r_{i p} r_{j q}\right] \partial_{p} \partial_{q}\left(-\Delta_{\perp}^{-1} \mathscr{A}\right)+\left(n_{i p} r_{j q}+n_{j p} r_{i q}\right) \partial_{p} \partial_{q}\left(-\Delta_{\perp}^{-1} \mathscr{B}\right) .
$$

\section{REFERENCES}

Alexandrova, O., Lacombe, C. and Mangeney, A. 2008 Spectra and anisotropy of magnetic fluctuations in the earths magnetosheath: Cluster observations. Ann. Geophys. 26, 3585-3596.

Borgogno, D., Passot, T. and Sulem, P. L. 2007 Magnetic holes in plasmas close to mirror instability. Nonlinear Proc. Geophys. 14, 373-383.

Cerri, S. S., Henri, P., Califano, F., Del Sarto, D., Faganello, M. and Pegoraro, F. 2013 Extended fluid models: Pressure tensor effects and equilibria. Phys. Plasmas 20, 112112. 
Goswami, P., Passot, T. and Sulem, P. L. 2005 A Landau fluid model for warm collisionless plasmas. Phys. Plasmas 12, 102109.

Hedrick, C. L. and Leboeuf, J. N. 1992 Landau fluid equations for electromagnetic and electrostatic fluctuations. Phys. Fluids B4, 3915-3934.

Howes, G. G., Cowley, S. C., Dorland, W., Hammett, G. W., E.Quataert and Schekochihin, A. A. 2006 Astrophysical gyrokinetics: Basic equations and linear theory. Astrophys. J. 651, 590-614.

Hunana, P., Goldstein, M. L., Passot, T., Sulem, P. L., Laveder, D. and Zank, G. P. 2013a Polarization and compressibility of oblique kinetic Alfvén waves. Astrophys. J. 766, 93.

Hunana, P., Goldstein, M. L., Passot, T., Sulem, P. L., Laveder, D. and Zank, G. P. 2013b Properties of kinetic Alfvén waves: A comparison of fluid models with kinetic theory. In: SOLAR WIND 13: Proc. 13th Int. Solar Wind Conference, AIP Conf. Proc., Vol. 1539, pp. 179-182.

Laveder, D., Marradi, L., Passot, T. and Sulem, P. L. 2011 Fluid simulations of mirror constraints on proton temperature anisotropy in solar wind turbulence. Geophys. Res. Lett. 38, L17108.

Laveder, D., Passot, T. and Sulem, P. L. 2013 Fluid simulations of non-resonant anisotropic ion heating. Ann. Geophys. 31, 1195-1204.

Macmahon, A. 1965 Finite gyro-radius corrections to the hydromagnetic equations for a Vlasov plasma. Phys. Fluids 8, 1840-1845.

Matteini, L., Hellinger, P., Landi, S., Trávníček, P. M. and Velli, M. 2012 Ion kinetics in the solar wind: Coupling global expansion to local microphysics. Space Sci. Rev. 172, 373-396.

Passot, T., Henri, P., Laveder, D. and Sulem, P. L. 2014 A fluid approach for ion scale plasmas with weakly distorted magnetic fields. Eur. Phys. J. D. 68, 207.

Passot, T. and Sulem, P. L. 2007 Collisionless magnetohydrodynamics with gyrokinetic effects. Phys. Plasmas 14, 082502.

Passot, T., Sulem, P. L. and Hunana, P. 2012 Extending magnetohydrodynamics to the slow dynamics of collisionless plasmas. Phys. Plasmas 19, 082113.

Ramos, J. J. 2005 Fluid formalism for collisionless magnetized plasmas. Phys. Plasmas 12, 052102.

Rönnmark, K. 1982 Waves in homogeneous, anisotropic multicomponent plasmas (WHAMP). Tech. Rep. 179. Kiruna Geophysical Institute.

Sahraoui, F., Goldstein, M. L., Belmont, G., Canu, P. and Rezeau, L. 2010 Three dimensional anisotropic $k$ spectra of turbulence at subproton scales in the solar wind. Phys. Rev. Lett. 105, 131101.

Sahraoui, F., Goldstein, M. L., Robert, P. and Khotyaintsev, Y. U. 2009 Evidence of a cascade and dissipation of solar-wind turbulence at the electron gyroscale. Phys. Rev. Lett. 102, 231102.

Schekochihin, A. A., Cowley, S. C., Rincon, F. and Rosin, M. S. 2010 Magnetofuid dynamics of magnetized cosmic plasma: Firehose and gyrothermal instabilities. Mon. Not. R. Astron. Soc. 405, 291-300.

Sharma, P., Hammett, G. W., Quataert, E. and Stone, J. M. 2006 Shearing box simulation of the MRI in a collisionless plasma. Astrophys. J. 637, 952-967.

Snyder, P. B., Hammett, G. W. and Dorland, W. 1997 Landau fluid models of collisionless magnetohydrodynamics. Phys. Plasmas 4, 3974-3985. 